\title{
High-Level Coupled-Cluster Energetics by Merging Moment Expansions with Selected Configuration Interaction
}

Accepted Manuscript: This article has been accepted for publication and undergone full peer review but has not been through the copyediting, typesetting, pagination, and proofreading process, which may lead to differences between this version and the Version of Record.

Cite as: J. Chem. Phys. (in press) (2021); https://doi.org/10.1063/5.0064400

Submitted: 22 July 2021 . Accepted: 11 October 2021 . Accepted manuscript online: 11 November 2021

Karthik Gururangan, (D) J. Emiliano Deustua, (D) Jun Shen, et al.

Challenge us.

What are your needs for periodic signal detection?

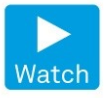

Zurich Instruments

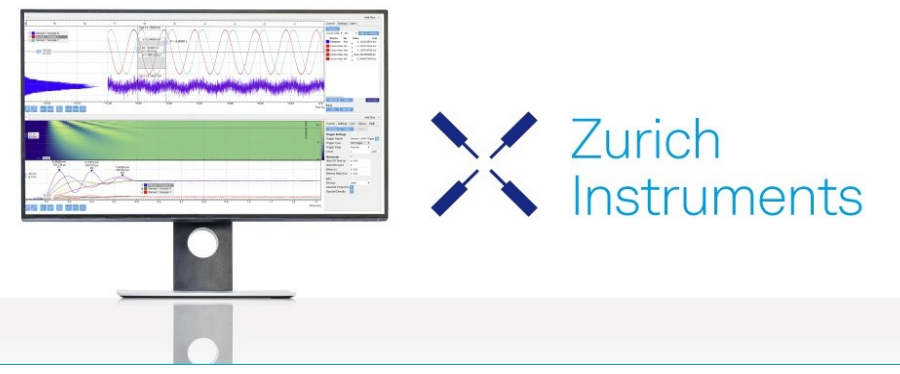




\title{
High-Level Coupled-Cluster Energetics by Merging Moment Expansions with Selected Configuration Interaction
}

\author{
Karthik Gururangan, ${ }^{1}$ J. Emiliano Deustua, ${ }^{1, a)}$ Jun Shen, ${ }^{1}$ and Piotr Piecuch ${ }^{1,2, b)}$ \\ ${ }^{1)}$ Department of Chemistry, Michigan State University, East Lansing, Michigan 48824, \\ USA \\ ${ }^{2)}$ Department of Physics and Astronomy, Michigan State University, East Lansing, Michigan 48824, \\ $U S A$
}

(Dated: 8 October 2021)

Inspired by our earlier semi-stochastic work aimed at converging high-level coupled-cluster (CC) energetics [J. E. Deustua, J. Shen, and P. Piecuch, Phys. Rev. Lett. 119, 223003 (2017); J. Chem. Phys. 154, $124103(2021)$, we propose a novel form of the $\mathrm{CC}(P ; Q)$ theory in which the stochastic Quantum Monte Carlo propagations, used to identify dominant higher-than-doubly excited determinants, are replaced by the selected configuration interaction (CI) approach using the CIPSI algorithm. The advantages of the resulting CIPSI-driven $\mathrm{CC}(P ; Q)$ methodology are illustrated by a few molecular examples, including the dissociation of $\mathrm{F}_{2}$ and the automerization of cyclobutadiene, where we recover the electronic energies corresponding to the $\mathrm{CC}$ calculations with a full treatment of singles, doubles, and triples based on the information extracted from compact CI wave functions originating from relatively inexpensive Hamiltonian diagonalizations.

\section{INTRODUCTION}

One of the key objectives of quantum chemistry is to obtain accurate energetics of molecular systems in a computationally efficient manner. Among the various post-Hartree-Fock (post-HF) theories, the size extensive approaches derived from the exponential ansatz ${ }^{1,2}$ of coupled-cluster (CC) theory ${ }^{3-7}$ are among the best techniques to accomplish this task. ${ }^{8,9}$ We recall that the $\mathrm{CC}$ wave function for an $N$-electron system is defined as

$$
|\Psi\rangle=e^{T}|\Phi\rangle,
$$

where $|\Phi\rangle$ is the reference (usually, HF) determinant and

$$
T=\sum_{n=1}^{N} T_{n}
$$

is the cluster operator, with $T_{n}$ representing its $n$-body ( $n$-particle- $n$-hole) component. In practice, one truncates the cluster operator $T$ at a given many-body rank to define the standard CC hierarchy of approximations. The most basic and most practical one, obtained when $T$ is truncated at $T_{2}$, which has computational steps that scale as $\mathcal{O}\left(\mathcal{N}^{6}\right)$ with the system size $\mathcal{N}$, is the CC method with singles and doubles (CCSD). ${ }^{10,11}$ The next two levels, namely, the CC approach with singles, doubles, and triples, abbreviated as CCSDT, ${ }^{12-14}$ which interests us in this study most, obtained when $T$ is truncated at $T_{3}$, and the CC method with singles, doubles, triples, and quadruples, abbreviated as CCSDTQ,${ }^{15-17}$ in which $T$ is truncated at $T_{4}$, involve the $\mathcal{O}\left(\mathcal{N}^{8}\right)$ and $\mathcal{O}\left(\mathcal{N}^{10}\right)$ steps,

\footnotetext{
a) Present address: Division of Chemistry and Chemical Engineering, California Institute of Technology, Pasadena, California 91125, USA.

b) Corresponding author; e-mail: piecuch@chemistry.msu.edu.
}

respectively. It is well established that in the majority of chemical applications, including molecules near equilibrium geometries, bond dissociations involving smaller numbers of strongly correlated electrons, noncovalent interactions, and photochemistry, the conventional CCSD, CCSDT, CCSDTQ, etc. hierarchy and its equation-ofmotion $(\mathrm{EOM})^{18-25}$ and linear-response ${ }^{26-34}$ extensions rapidly approach the exact, full configuration interaction (FCI) limit, so that by the time one reaches the CCSDT or CCSDTQ levels, the results are usually converged with respect to the relevant many-electron correlation effects, ${ }^{9}$ but the $\mathcal{O}\left(\mathcal{N}^{8}\right)$ computational steps of CCSDT or the $\mathcal{O}\left(\mathcal{N}^{10}\right)$ steps of CCSDTQ render the usage of such methods unfeasible for most problems of interest. Thus, one of the main activities in the $\mathrm{CC}$ development work has been the design of high-fidelity approximations to CCSDT and CCSDTQ, capable of reducing the above costs, while being more robust than perturbative approaches of the $\operatorname{CCSD}(\mathrm{T})^{35}$ type, which fail in more multi-reference situations..$^{8,9,36-38}$

To that end, our group has recently developed the semi-stochastic $\mathrm{CC}(P ; Q)$ formalism, ${ }^{39-41}$ a novel methodology that can efficiently converge the energetics of high-level CC calculations, such as CCSDT, CCSDTQ, and EOMCCSDT, ${ }^{21-23}$ by combining the deterministic $\mathrm{CC}(P ; Q)$ framework ${ }^{42-46}$ with the stochastic Quantum Monte Carlo (QMC) wave function propagations in the many-electron Hilbert space defining the CIQMC ${ }^{47-51}$ and $\mathrm{CC}$ Monte Carlo (CCMC) $)^{52-55}$ approaches. The semi-stochastic $\mathrm{CC}(P ; Q)$ methodology of Refs. 39-41 leverages the fact, recognized long time ago in the context of active-space CC considerations (cf. Ref. 38 for a review), that higher-order cluster operators, such as $T_{3}$ and $T_{4}$, and their counterparts utilized in EOMCC are usually relatively sparse. In the semi-stochastic $\mathrm{CC}(P ; Q)$ approaches, the dominant higher-than-doubly excited cluster/excitation amplitudes relevant to the parent CC/EOMCC theory of interest are automatically se- 
lected using stochastic CIQMC or CCMC wave function propagations that provide lists of Slater determinants for the initial $\mathrm{CC}(P)^{39,40}$ or $\operatorname{EOMCC}(P)^{41,56}$ calculations, which are subsequently corrected using the biorthogonal moment expansions adopted in the $\mathrm{CC}(P ; Q)$ formalism to capture the remaining correlations. The semistochastic $\mathrm{CC}(P ; Q)$ methods have demonstrated their ability to rapidly converge the CCSDT, ${ }^{39-41}$ CCSDTQ, ${ }^{40}$ and EOMCCSDT ${ }^{41}$ energetics out of the early stages of the underlying CIQMC or CCMC propagations, with minimal reliance on user- and system-dependent inputs.

Encouraged by the above findings, in this study we explore the use of selected CI as an alternative provider of the lists of the leading higher-than-doubly excited determinants needed to drive the $\mathrm{CC}(P ; Q)$ computations. The selected CI schemes, which date back to the pioneering efforts in the late 1960s and early $1970 \mathrm{~s},{ }^{57-60}$ have recently regained significant interest, as their modern implementations have demonstrated the ability to capture the bulk of many-electron correlation effects in a computationally efficient manner using a conceptually straightforward linear wave function ansatz. ${ }^{61-73}$ The selected CI model adopted in the $\mathrm{CC}(P ; Q)$ considerations reported in this work is the CI method using perturbative selection made iteratively (CIPSI), ${ }^{59}$ as recently reformulated and further developed in Refs. 70 and 71.

\section{THEORY AND ALGORITHMIC DETAILS}

We begin by reviewing the key elements of the groundstate $\mathrm{CC}(P ; Q)$ formalism relevant to this work. Each $\mathrm{CC}(P ; Q)$ calculation starts by identifying two disjoint subspaces of the $N$-electron Hilbert space, the $P$ space designated as $\mathscr{H}^{(P)}$ and the $Q$ space denoted as $\mathscr{H}^{(Q)}$. The former space is spanned by the excited determinants $\left|\Phi_{K}\right\rangle=E_{K}|\Phi\rangle$, where $E_{K}$ is the elementary particle-hole excitation operator generating $\left|\Phi_{K}\right\rangle$ from $|\Phi\rangle$, which together with $|\Phi\rangle$ dominate the ground-state wave function, whereas the determinants in $\mathscr{H}^{(Q)}$ are used to construct the correction due to the correlation effects the $\mathrm{CC}$ calculations in the $P$ space do not describe. Once the $P$ and $Q$ spaces are defined, we solve the $\mathrm{CC}$ amplitude equations

$$
\mathfrak{M}_{K}(P)=0,\left|\Phi_{K}\right\rangle \in \mathscr{H}^{(P)},
$$

where

$$
\mathfrak{M}_{K}(P)=\left\langle\Phi_{K}\left|\bar{H}^{(P)}\right| \Phi\right\rangle
$$

with

$$
\bar{H}^{(P)}=e^{-T^{(P)}} H e^{T^{(P)}},
$$

are moments of the CC equations, ${ }^{74-76}$ to obtain the approximate form of the cluster operator in the $P$ space,

$$
T^{(P)}=\sum_{\left|\Phi_{K}\right\rangle \in \mathscr{H}^{(P)}} t_{K} E_{K}
$$

and the corresponding ground-state energy

$$
E^{(P)}=\left\langle\Phi\left|\bar{H}^{(P)}\right| \Phi\right\rangle,
$$

and calculate the noniterative correction $\delta(P ; Q)$ to determine the final $\mathrm{CC}(P ; Q)$ energy as

$$
E^{(P+Q)}=E^{(P)}+\delta(P ; Q) .
$$

The correction $\delta(P ; Q)$ to the energy $E^{(P)}$ obtained in the $P$-space $\mathrm{CC}[\mathrm{CC}(P)]$ calculations is given by ${ }^{42,43}$

$$
\delta(P ; Q)=\sum_{\left|\Phi_{K}\right\rangle \in \mathscr{H}^{(Q)}} \ell_{K}(P) \mathfrak{M}_{K}(P),
$$

where $\mathfrak{M}_{K}(P)$ is defined by Eq. (4) and

$$
\ell_{K}(P)=\left\langle\Phi\left|\left(1+\Lambda^{(P)}\right) \bar{H}^{(P)}\right| \Phi_{K}\right\rangle / D_{K}^{(P)},
$$

with

$$
D_{K}^{(P)}=E^{(P)}-\left\langle\Phi_{K}\left|\bar{H}^{(P)}\right| \Phi_{K}\right\rangle
$$

The $\Lambda^{(P)}$ operator entering Eq. (10), given by

$$
\Lambda^{(P)}=\sum_{\left|\Phi_{K}\right\rangle \in \mathscr{H}^{(P)}} \lambda_{K}\left(E_{K}\right)^{\dagger}
$$

and obtained by solving the linear system

$$
\left\langle\Phi\left|\left(1+\Lambda^{(P)}\right) \bar{H}^{(P)}\right| \Phi_{K}\right\rangle=E^{(P)} \lambda_{K}, \quad\left|\Phi_{K}\right\rangle \in \mathscr{H}^{(P)}
$$

is the hole-particle deexcitation operator defining the bra state $\left\langle\tilde{\Psi}^{(P)}\right|=\langle\Phi|\left(1+\Lambda^{(P)}\right) e^{-T^{(P)}}$ associated with the $\mathrm{CC}(P)$ ket state $\left|\Psi^{(P)}\right\rangle=e^{T^{(P)}}|\Phi\rangle$.

The $\mathrm{CC}(P ; Q)$ formalism includes the completely renormalized CC methods, such as $\mathrm{CR}-\mathrm{CC}(2,3),{ }^{77-80}$ which works better than $\operatorname{CCSD}(\mathrm{T})$ in bond breaking situations, but its main advantage is the freedom to make unconventional choices of the $P$ and $Q$ spaces, allowing one to relax the lower-order $T_{1}$ and $T_{2}$ clusters in the presence of their higher-order counterparts, such as the leading $T_{3}$ contributions, which the $\operatorname{CCSD}(\mathrm{T}), \mathrm{CR}-\mathrm{CC}(2,3)$, and other triples corrections to CCSD cannot do. One can use active orbitals to identify the leading higher-than-doubly excited determinants for the inclusion in the $P$ space used in the $\mathrm{CC}(P)$ calculations, employing the $\delta(P ; Q)$ corrections to capture the remaining correlations of interest, as in the $\mathrm{CC}(\mathrm{t} ; 3)$ and similar approaches, ${ }^{42-46}$ or adopt a more black-box semi-stochastic $\mathrm{CC}(P ; Q)$ framework, in which the selection of the dominant higher-than-doubly excited determinants entering the $P$ space is automated with the help of CIQMC or CCMC propagations. ${ }^{39-41}$ In this article, we propose an alternative to the semistochastic $\mathrm{CC}(P ; Q)$ methodology, in which we use the information extracted from the CIPSI runs to populate the $P$ spaces employed in the $\mathrm{CC}(P)$ calculations preceding the determination of the $\delta(P ; Q)$ corrections. 
We recall that the CIPSI approach, originally proposed in Ref. 59 and further developed in Refs. 70 and 71 , seeks to construct an approximation to the FCI wave function by a series of Hamiltonian diagonalizations in increasingly large, iteratively defined, subspaces of the many-electron Hilbert space, designated as $\mathcal{V}_{\text {int }}^{(k)}$, where $k=0,1,2, \ldots$ enumerates the consecutive CIPSI iterations. The initial subspace $\mathcal{V}_{\text {int }}^{(0)}$ can be onedimensional, if the CIPSI calculations are started from a single determinant, such as the restricted HF (RHF) wave function used throughout this work as a reference, or multi-dimensional, if one prefers to start from a multideterminantal state generated in some preliminary truncated CI computation, and the remaining subspaces are constructed via a recursive process in which $\mathcal{V}_{\text {int }}^{(k+1)}$ is obtained by augmenting $\mathcal{V}_{\text {int }}^{(k)}$ with a subset of the leading singly and doubly excited determinants out of $\mathcal{V}_{\text {int }}^{(k)}$ identified with the help of the many-body perturbation theory (MBPT). Thus, if $\left|\Psi_{k}^{(\mathrm{CIPSI})}\right\rangle=\sum_{\left|\Phi_{I}\right\rangle \in \mathcal{V}_{\text {int }}^{(k)}} c_{I}\left|\Phi_{I}\right\rangle$ is a CI wave function obtained by diagonalizing the Hamiltonian in the current subspace $\mathcal{V}_{\text {int }}^{(k)}$ and $E_{\mathrm{var}, k}$ is the corresponding energy, and if $\mathcal{V}_{\text {ext }}^{(k)}$ is the space of all singly and doubly excited determinants out of $\left|\Psi_{k}^{(\mathrm{CIPSI})}\right\rangle$, for each determinant $\left|\Phi_{\alpha}\right\rangle \in \mathcal{V}_{\text {ext }}^{(k)}$ we evaluate the second-order MBPT correction $e_{\alpha, k}^{(2)}=\left|\left\langle\Phi_{\alpha}|H| \Psi_{k}^{(\mathrm{CIPSI})}\right\rangle\right|^{2} /\left(E_{\mathrm{var}, k}-\right.$ $\left.\left\langle\Phi_{\alpha}|H| \Phi_{\alpha}\right\rangle\right)$ and use the resulting $e_{\alpha, k}^{(2)}$ values to decide which determinants from $\mathcal{V}_{\text {ext }}^{(k)}$ should be added to the determinants $\left|\Phi_{I}\right\rangle$ already in $\mathcal{V}_{\text {int }}^{(k)}$ to construct the next diagonalization space $\mathcal{V}_{\text {int }}^{(k+1)}$. We can also use the $e_{\alpha, k}^{(2)}$ values to calculate the perturbatively corrected CIPSI energies $E_{\mathrm{var}, k}+\Delta E_{k}^{(2)}$, where $\Delta E_{k}^{(2)}=\sum_{\left|\Phi_{\alpha}\right\rangle \in \mathcal{V}_{\text {ext }}^{(k)}} e_{\alpha, k}^{(2)}$, and, after further manipulations, their $E_{\mathrm{var}, k}+\Delta E_{\mathrm{r}, k}^{(2)}$ counterparts, in which $\Delta E_{k}^{(2)}$ is replaced by its renormalized $\Delta E_{\mathrm{r}, k}^{(2)}$ form introduced in Ref. 71.

In the modern implementation of CIPSI, formulated in Refs. 70 and 71 and available in the Quantum Package 2.0 software, ${ }^{71}$ which we used in the present study, the process of enlarging $\mathcal{V}_{\text {int }}^{(k)}$ to generate $\mathcal{V}_{\text {int }}^{(k+1)}$ is executed in the following manner. First, prior to examining the $e_{\alpha, k}^{(2)}$ corrections, one stochastically samples the most important singly and doubly excited determinants out of $\left|\Psi_{k}^{(\mathrm{CIPSI})}\right\rangle$, so that not all singles and doubles from $\mathcal{V}_{\text {int }}^{(k)}$ are included in the accompanying $\mathcal{V}_{\text {ext }}^{(k)}$ space, only the sampled ones. Next, one arranges the sampled determinants $\left|\Phi_{\alpha}\right\rangle \in \mathcal{V}_{\text {ext }}^{(k)}$ in descending order according to their $\left|e_{\alpha, k}^{(2)}\right|$ values. The process of enlarging the current subspace $\mathcal{V}_{\text {int }}^{(k)}$ to construct the $\mathcal{V}_{\text {int }}^{(k+1)}$ space for the subsequent Hamiltonian diagonalization, which starts from the determinants $\left|\Phi_{\alpha}\right\rangle$ characterized by the largest $\left|e_{\alpha, k}^{(2)}\right|$ contributions, moving toward those that have smaller $\left|e_{\alpha, k}^{(2)}\right|$ values, continues until the number of determinants in $\mathcal{V}_{\text {int }}^{(k+1)}$ exceeds the dimension of $\mathcal{V}_{\text {int }}^{(k)}$ multiplied by a user-defined factor $f>1$. In this study, we used $f=2$, which is the default in Quantum Package 2.0 (we will examine other choices of $f$ in the future). In practice, a typical dimension of $\mathcal{V}_{\mathrm{int}}^{(k+1)}$, including each of the final diagonalization spaces used to generate lists of higherthan-doubly excited determinants for the $\mathrm{CC}(P)$ calculations reported in this work, is slightly larger than $f$ times the dimension of $\mathcal{V}_{\mathrm{int}}^{(k)}$, since the CIPSI algorithm adds extra determinants to $\mathcal{V}_{\text {int }}^{(k+1)}$ to ensure that the resulting $\left|\Psi_{k+1}^{(\mathrm{CIPSI})}\right\rangle$ wave function is an eigenstate of the total spin $S^{2}$ and $S_{z}$ operators. The final wave function $\left|\Psi^{\text {(CIPSI })}\right\rangle$ of a given CIPSI run and the associated variational $\left(E_{\mathrm{var}}\right)$ and perturbatively corrected $\left[E_{\mathrm{var}}+\Delta E^{(2)}\right.$ and $\left.E_{\mathrm{var}}+\Delta E_{\mathrm{r}}^{(2)}\right]$ energies are obtained by terminating the above procedure in one of the following two ways: (i) stopping at the first iteration $k$ for which the secondorder MBPT correction $\left|\Delta E_{k}^{(2)}\right|$ falls below a user-defined threshold $\eta$, indicating that the CIPSI wave function is within a tolerable distance from FCI, or (ii) stopping at the first iteration $k$ for which the number of determinants in the corresponding $\mathcal{V}_{\text {int }}^{(k)}$ space exceeds a user-defined input parameter $N_{\operatorname{det}(\text { in) }}$. Since our main objective is to employ the CIPSI-driven $\mathrm{CC}(P ; Q)$ algorithm to accurately reproduce the high-level CC rather than FCI energetics, without having to converge the underlying CIPSI sequence, we chose the latter option, which we enforced by using $\eta=10^{-6}$ hartree. As a result of setting the input parameter $f$ at 2, the sizes of the final wave functions $\left|\Psi^{(\mathrm{CIPSI})}\right\rangle$ produced by our CIPSI runs, denoted as $N_{\operatorname{det}(\text { out })}$, were always between $N_{\operatorname{det}(\text { in) }}$ and $2 N_{\operatorname{det}(\text { in) }}$.

Having discussed the key ingredients of the $\operatorname{CC}(P ; Q)$ and CIPSI methodologies relevant to this work, we proceed to the description of the CIPSI-driven $\operatorname{CC}(P ; Q)$ algorithm, which consists of the following steps:

1. Given a reference state $|\Phi\rangle$, which in all of the calculations reported in this article was the RHF determinant, choose an input parameter $N_{\operatorname{det}(\text { in) }}$, used to terminate the CIPSI wave function growth, and execute a CIPSI run to obtain the final state $\left|\Psi^{(\mathrm{CIPSI})}\right\rangle$ spanned by $N_{\text {det(out) }}$ determinants.

2. Extract a list of higher-than-doubly excited determinants relevant to the desired CC theory level from $\left|\Psi^{(\mathrm{CIPSI})}\right\rangle$ to define the $P$ space. If the goal is to converge the CCSDT energetics, the $P$ space consists of all singly and doubly excited determinants plus the triply excited determinants contained in $\left|\Psi^{(\mathrm{CIPSI})}\right\rangle$. To recover the CCSDTQ energetics, quadruply excited determinants contributing to $\left|\Psi^{(\mathrm{CIPSI})}\right\rangle$ are included in the $P$ space as well.

3. Solve the nonlinear $\mathrm{CC}(P)$ system, Eq. (3), and the associated linear system given by Eq. (13), where $E^{(P)}$ is defined by Eq. (7), in the $P$ space determined in Step 2 to obtain the cluster operator $T^{(P)}$ and the deexcitation operator $\Lambda^{(P)}$. If 
the target approach is CCSDT, define $T^{(P)}=T_{1}+$ $T_{2}+T_{3}^{(\mathrm{CIPSI})}$ and $\Lambda^{(P)}=\Lambda_{1}+\Lambda_{2}+\Lambda_{3}^{(\mathrm{CIPSI})}$, where the list of triples entering $T_{3}^{\text {(CIPSI) }}$ and $\Lambda_{3}^{\text {(CIPSI) }}$ is identical to that extracted from $\left|\Psi^{(\mathrm{CIPSI})}\right\rangle$ in Step 2 . If the goal is to converge the CCSDTQ energetics, define $T^{(P)}=T_{1}+T_{2}+T_{3}^{(\mathrm{CIPSI})}+T_{4}^{(\mathrm{CIPSI})}$ and $\Lambda^{(P)}=\Lambda_{1}+\Lambda_{2}+\Lambda_{3}^{(\mathrm{CIPSI})}+\Lambda_{4}^{(\mathrm{CIPSI})}$, where the list of triples entering $T_{3}^{(\mathrm{CIPSI})}$ and $\Lambda_{3}^{(\mathrm{CIPSI})}$ and the list of quadruples entering $T_{4}^{\text {(CIPSI) }}$ and $\Lambda_{4}^{\text {(CIPSI) }}$ are again extracted from $\left|\Psi^{(\text {CIPSI })}\right\rangle$.

4. Use the information obtained in Step 3 to determine correction $\delta(P ; Q)$, Eq. (9), which describes the remaining correlations of interest that were not captured by the $\mathrm{CC}(P)$ calculations. If the goal is to converge the CCSDT energetics, define the $Q$ space needed to calculate $\delta(P ; Q)$ as the remaining triply excited determinants that are not contained in $\left|\Psi^{(\mathrm{CIPSI})}\right\rangle$. If the target approach is CCSDTQ, define the $Q$ space as the triply and quadruply excited determinants absent in $\left|\Psi^{(\mathrm{CIPSI})}\right\rangle$. Add the resulting correction $\delta(P ; Q)$ to $E^{(P)}$ to obtain the $\mathrm{CC}(P ; Q)$ energy $E^{(P+Q)}$, Eq. (8).

5. To check convergence, repeat Steps 1-4 for a larger value of $N_{\operatorname{det}(\text { in) }}$. The CIPSI-driven $\mathrm{CC}(P ; Q)$ calculations can be regarded as converged if the difference between consecutive $E^{(P+Q)}$ energies falls below a user-defined threshold. In analogy to the semi-stochastic $\operatorname{CC}(P ; Q)$ framework of Refs. 39-41, one can also stop if the fraction(s) of higher-thandoubly excited determinants contained in the final CIPSI state $\left|\Psi^{(\mathrm{CIPSI})}\right\rangle$ is (are) sufficiently large to produce the desired accuracy level.

In this initial exploratory study, we implemented the CIPSI-driven $\mathrm{CC}(P ; Q)$ approach that allows us to converge the CCSDT energetics. We did this by modifying our standalone $\operatorname{CC}(P ; Q)$ codes, described in Refs. 39-45 and interfaced with the RHF and integral transformation routines available in GAMESS, ${ }^{81,82}$ such that they can use the lists of triply excited determinants extracted from the CIPSI wave functions $\left|\Psi^{(\mathrm{CIPSI})}\right\rangle$, generated with Quantum Package 2.0, to set up the relevant $P$ spaces (as already explained, the corresponding $Q$ spaces are automatically defined as the remaining triples absent in the $\left|\Psi^{\text {(CIPSI) }}\right\rangle$ wave functions). By design, as the input parameter $N_{\operatorname{det}(i n)}$ used to terminate CIPSI runs increases, producing longer and longer CI expansions to represent wave functions $\left|\Psi^{(\mathrm{CIPSI})}\right\rangle$, the $\mathrm{CC}(P ; Q)$ energies $E^{(P+Q)}$ approach their CCSDT parents. The underlying $\mathrm{CC}(P)$ calculations converge the CCSDT energetics too, but, as further elaborated on in Section III, by ignoring the triples that were not captured by CIPSI, they do it at a much slower rate. In examining the convergence of the CIPSI-driven $\mathrm{CC}(P)$ and $\operatorname{CC}(P ; Q)$ energies toward CCSDT, we sampled the $N_{\operatorname{det}(i n)}$ values in a roughly semi-logarithmic manner, starting from $N_{\operatorname{det}(\text { in })}=1$. Since all of the calculations reported in this work adopted RHF determinants as reference functions, the $\left|\Psi^{(\mathrm{CIPSI})}\right\rangle$ state becomes the RHF determinant and the resulting $\mathrm{CC}(P)$ and $\mathrm{CC}(P ; Q)$ energies become identical to those obtained in the RHF-based CCSD and CR-CC $(2,3)$ calculations, respectively, when $N_{\operatorname{det}(\text { in })}=1$. Thus, in analogy to the QMC propagation time $\tau$ used in our semi-stochastic $\mathrm{CC}(P) / \operatorname{EOMCC}(P)$ and $\operatorname{CC}(P ; Q)$ studies, ${ }^{39-41,56}$ we can regard the $N_{\operatorname{det}(\text { in })}$ input variable defining CIPSI computations as the parameter connecting $\mathrm{CCSD}$ [in the $\mathrm{CC}(P)$ case] or CR$\mathrm{CC}(2,3)$ [in the case of $\mathrm{CC}(P ; Q)$ runs] with CCSDT. As a result, similarly to CCSD, CR-CC $(2,3)$, and CCSDT, the CIPSI-driven $\mathrm{CC}(P)$ and $\mathrm{CC}(P ; Q)$ approaches considered in this work remain size extensive for all values of $N_{\operatorname{det}(\text { in) }}$. The $\mathrm{CC}(P)$ calculations are size extensive, since they are nothing else than the usual CC computations in which we solve the connected amplitude equations, Eq. (3), for the cluster operator $T^{(P)}$ defined by Eq. (6). In the case of the CIPSI-driven $\mathrm{CC}(P)$ method implemented in this study, $T^{(P)}=T_{1}+T_{2}+T_{3}^{(\mathrm{CIPSI})}$, where $T_{3}^{(\mathrm{CIPSI})}=\sum_{\left|\Phi_{i j k}^{a b c}\right\rangle \in\left|\Psi^{(\mathrm{CIPSI})}\right\rangle} t_{a b c}^{i j k} E_{i j k}^{a b c}$ is the $T_{3}$ operator defined using the list of triply excited determinants $\left|\Phi_{i j k}^{a b c}\right\rangle$ contained in the final CIPSI state $\left|\Psi^{(\mathrm{CIPSI})}\right\rangle$ (we use the usual notation in which $i, j, k$ and $a, b, c$ designate the occupied and unoccupied spin-orbitals in $|\Phi\rangle$, respectively, and $E_{i j k}^{a b c}$ is the elementary triple excitation operator generating $\left|\Phi_{i j k}^{a b c}\right\rangle$ from $\left.|\Phi\rangle\right)$. The noniterative correction $\delta(P ; Q)$, Eq. (9), which in the case of the CIPSI-driven $\mathrm{CC}(P ; Q)$ approach developed in this work captures the $T_{3}$ effects not described by $T_{3}^{\text {(CIPSI) }}$ and which involves the summation over the remaining triply excited determinants that are not included in $\left|\Psi^{(\mathrm{CIPSI})}\right\rangle$, i.e., $\delta(P ; Q)=\sum_{\left|\Phi_{i j k}^{a b c}\right\rangle \notin\left|\Psi_{(\mathrm{CIPSI})}\right\rangle} \ell_{i j k}^{a b c} \mathfrak{M}_{a b c}^{i j k}$, being the connected quantity similar to that used in the CR-CC $(2,3)$ and $\mathrm{CC}(\mathrm{t} ; 3)$ methods, is size extensive too (for the early numerical illustration of the size extensivity of CR-CC(2,3), see Ref. 78).

The numerical demonstration of the size extensivity of the CIPSI-driven $\mathrm{CC}(P)$ and $\mathrm{CC}(P ; Q)$ methods implemented in this work is shown in Table I. Our example is the noninteracting $\mathrm{F}_{2}+\mathrm{Ne}$ system, described by the cc-pVDZ basis set, ${ }^{83}$ obtained by placing the Ne atom at 1,000 bohr from the stretched fluorine molecule in which the $\mathrm{F}-\mathrm{F}$ bond length was set at twice its equilibrium value to increase the magnitude of $T_{3}$ correlations. Along with the $\mathrm{F}_{2}+\mathrm{Ne}$ system, we consider the isolated $\mathrm{F}_{2}$ molecule having the same geometry as in $\mathrm{F}_{2}+\mathrm{Ne}$ and the isolated neon atom, both described by the cc-pVDZ basis. The CIPSI diagonalization sequence used to provide the list of triply excited determinants for the inclusion in the $P$ space corresponding to the $\mathrm{F}_{2}+\mathrm{Ne}$ system was initiated from the RHF reference determinant and defined by setting the wave function termination parameter $N_{\operatorname{det}(\text { in) }}$, the input parameter $f$ controlling the CIPSI wave function growth, and the MBPT-based stopping parameter $\eta$ at $5,000,2$, and $10^{-6}$ hartree, respectively. Following the 
above description, the $P$ space used in the CIPSI-driven $\mathrm{CC}(P)$ calculation for the noninteracting $\mathrm{F}_{2}+$ Ne dimer consisted of all singly and doubly excited determinants and a subset of triply excited determinants contained in the last $\left|\Psi^{(\mathrm{CIPSI})}\right\rangle$ state of the $N_{\operatorname{det}(\mathrm{in})}=5,000$ CIPSI run. The $Q$ space needed to compute the corresponding $\mathrm{CC}(P ; Q)$ correction $\delta(P ; Q)$ was defined as the remaining triples not included in $\left|\Psi^{\text {(CIPSI) }}\right\rangle$. To ensure the consistency of the $P$ spaces used in the $\mathrm{CC}(P)$ calculations for the $\mathrm{F}_{2}+\mathrm{Ne}$ system and the $\mathrm{F}_{2}$ and Ne fragments, we generated the $P$ space for $\mathrm{F}_{2}$ by removing the triply excited determinants involving the orbitals of $\mathrm{Ne}$ from the list of triples obtained in the $N_{\operatorname{det}(\text { in) }}=5,000$ CIPSI run for $\mathrm{F}_{2}+\mathrm{Ne}$. Similarly, the $P$ space used in the $\mathrm{CC}(P)$ calculations for Ne was obtained by starting from the list of triples produced in the $N_{\operatorname{det}(i n)}=5,000$ CIPSI calculation for $\mathrm{F}_{2}+\mathrm{Ne}$ and removing the triply excited determinants involving the orbitals of $\mathrm{F}_{2}$. As in all $\mathrm{CC}(P ; Q)$ calculations considered in this work, the $Q$ spaces associated with the $\mathrm{F}_{2}$ and $\mathrm{Ne}$ monomers were defined as the remaining triples missing in the respective $P$ spaces. We chose the $N_{\operatorname{det}(i n)}=5,000$ value in the size extensivity test reported in Table I, since it is sufficiently large to introduce the leading triply excited determinants into the relevant $P$ spaces, while being small enough to produce the $\mathrm{CC}(P)$ and $\mathrm{CC}(P ; Q)$ energies that are visibly different than their CCSDT counterparts.

It is clear from the results presented in Table I that, in analogy to CCSD, CR-CC $(2,3)$, and CCSDT, the CIPSIdriven $\mathrm{CC}(P)$ and $\mathrm{CC}(P ; Q)$ methods are size extensive. Indeed, the $\mathrm{CC}(P)$ and $\mathrm{CC}(P ; Q)$ energies of the $\mathrm{F}_{2}+\mathrm{Ne}$ dimer are numerically identical to the corresponding sums of the energies of the $\mathrm{F}_{2}$ and Ne monomers. We observe the same behavior for other values of the CIPSI wave function termination parameter $N_{\operatorname{det}(i n)}$. One may ask a question why the interfragment triply excited determinants $\left|\Phi_{i j k}^{a b c}\right\rangle$ having spin-orbital indices located on different monomers, which are present in the final CIPSI state $\left|\Psi^{(\mathrm{CIPSI})}\right\rangle$ of the noninteracting $\mathrm{F}_{2}+\mathrm{Ne}$ system and thus end up in the corresponding $P$ space, do not result in the violation of size extensivity in the $\mathrm{CC}(P)$ and $\mathrm{CC}(P ; Q)$ calculations. The answer to this question is that the connected triply excited cluster amplitudes $t_{a b c}^{i j k}$ carrying indices located on different noninteracting fragments vanish when obtained by solving the explicitly connected $\mathrm{CC}(P)$ amplitude equations, Eq. (3). We did not remove such interfragment $t_{a b c}^{i j k}$ amplitudes from our $\mathrm{CC}(P)$ calculations for the $\mathrm{F}_{2}+$ Ne system and confirmed that they do indeed vanish. The use of CI diagonalizations in constructing the $P$ spaces for the $\mathrm{CC}(P)$ and $\mathrm{CC}(P ; Q)$ computations does not affect the size extensivity of the CIPSI-driven $\mathrm{CC}(P)$ and $\mathrm{CC}(P ; Q)$ approaches, since all we need from these diagonalizations are the lists of higher-than-doubly excited determinants relevant to the $\mathrm{CC}$ theory of interest (in our case, where we target the CCSDT energetics, the lists of triples), not the CI excitation amplitudes themselves. For example, as in all conventional CC calculations, the contributions from the interfragment triply excited determinants $\left|\Phi_{i j k}^{a b c}\right\rangle$ to the ground-state wave function of the noninteracting $\mathrm{F}_{2}+$ Ne dimer are represented in the $\mathrm{CC}(P)$ calculations by the disconnected $T_{1} T_{2}$ and $(1 / 6) T_{1}^{3}$ clusters. The noniterative correction $\delta(P ; Q)$, which provides information about those $T_{3}$ correlations that were not captured by the preceding $\mathrm{CC}(P)$ run, becomes the sum of the $\delta(P ; Q)$ values for the isolated $\mathrm{F}_{2}$ and $\mathrm{Ne}$ fragments.

Aside from size extensivity, as analyzed above, and high efficiency in converging the parent CCSDT energetics discussed in Section III, and in analogy to the activeorbital-based $^{42-45}$ and semi-stochastic ${ }^{39-41} \mathrm{CC}(P ; Q)$ approaches, the CIPSI-driven $\mathrm{CC}(P ; Q)$ methodology examined in this work offers significant savings in the computational effort compared to full CCSDT. This is largely related to the fact that, as shown in Section III, the convergence of the CIPSI-driven $\mathrm{CC}(P ; Q)$ energies toward their CCSDT parents with the wave function termination parameter $N_{\operatorname{det}(i n)}$, with the number of determinants used to generate the final CIPSI state $N_{\operatorname{det}(\text { out })}$, and with the fractions of triples in the $P$ space captured by the CIPSI algorithm is very fast. Indeed, the CPU times associated with the CIPSI runs using smaller $N_{\text {det(in) }}$ values, resulting in smaller diagonalization spaces, are relatively short compared to the converged CIPSI computations. Next, as explained in detail in Refs. 39-41, the $\mathrm{CC}(P)$ calculations using small fractions of triples in the $P$ space, which is all one needs to converge the CCSDT-level energetics in the CIPSI-driven $\mathrm{CC}(P ; Q)$ runs, are much faster than the corresponding CCSDT computations. Finally, as also explained in Refs. 39-41, the computational cost of determining the $\mathrm{CC}(P ; Q)$ correction $\delta(P ; Q)$ is less than the cost of a single iteration of CCSDT.

In examining the CIPSI-driven $\mathrm{CC}(P)$ and $\mathrm{CC}(P ; Q)$ energies in Section III, we are primarily interested in how fast they converge toward their parent CCSDT values as $N_{\text {det(in) }}$ and the fraction of triples in the $P$ space increase. In the case of the $E_{\mathrm{var}}, E_{\mathrm{var}}+\Delta E^{(2)}$, and $E_{\mathrm{var}}+\Delta E_{\mathrm{r}}^{(2)}$ energies, we do what is often done in CIPSI calculations (see, e.g., Refs. 71 and 72) and compare them to their counterparts obtained by extrapolating the data obtained in the CIPSI runs defined by the largest $N_{\operatorname{det}(i n)}$ values to the FCI limit. Specifically, following the procedure used in Ref. 72, we performed a linear fit of the last four $E_{\mathrm{var}, k}+\Delta E_{\mathrm{r}, k}^{(2)}$ energies leading to the final $\left|\Psi^{(\mathrm{CIPSI})}\right\rangle$ state obtained for the largest value of $N_{\operatorname{det}(i n)}$ in a given CIPSI sequence, plotted against the corresponding $\Delta E_{\mathrm{r}, k}^{(2)}$ corrections, and extrapolated the resulting line to the $\Delta E_{\mathrm{r}, k}^{(2)}=0$ limit.

\section{NUMERICAL EXAMPLES}

We illustrate potential benefits offered by the CIPSIdriven $\mathrm{CC}(P ; Q)$ methodology, when applied to recovering the CCSDT energetics, using a few molecular exam- 
ples. Our first example is the frequently studied dissociation of the fluorine molecule, as described by the cc-pVDZ basis set. We chose this example, since it is well established that the CCSDT approach provides an accurate description of bond breaking in $\mathrm{F}_{2}$ (cf., e.g., Refs. 77, 78, 84, and 85) and since we previously used it to benchmark the $\mathrm{CC}(P ; Q)$-based $\mathrm{CC}(\mathrm{t} ; 3)$ approach $^{42}$ and the semi-stochastic $\mathrm{CC}(P ; Q)$ methods driven by CIQMC $^{39,40}$ and CCMC $^{39}$ propagations. The results of our calculations for the $\mathrm{F}_{2}$ /cc-pVDZ system, in which the $\mathrm{F}-\mathrm{F}$ bond length $R$ was stretched from its equilibrium, $R_{e}=2.66816$ bohr, value, where electron correlation effects are largely dynamical in nature, to $1.5 R_{e}$, $2 R_{e}$, and $5 R_{e}$, where they gain an increasingly nondynamical character, are summarized in Table II and Fig. 1. The complexity of electron correlations in $\mathrm{F}_{2}$ manifests itself in the rapidly growing magnitude of $T_{3}$ contributions as the $\mathrm{F}-\mathrm{F}$ distance increases, as exemplified by the unsigned differences between the CCSDT and CCSD energies, which are 9.485 millihartree at $R=R_{e}$, 32.424 millihartree at $R=1.5 R_{e}, 45.638$ millihartree at $R=2 R_{e}$, and 49.816 millihartree at $R=5 R_{e}$, when the cc-pVDZ basis set is employed. They grow with $R$ so fast that in the $R=2 R_{e}-5 R_{e}$ region they become larger than the depth of the CCSDT potential (estimated at $\sim 44$ millihartree when the CCSDT energy at $R=R_{e}$ is subtracted from its $R=5 R_{e}$ counterpart) and highly nonperturbative. The latter feature of $T_{3}$ contributions in the stretched $\mathrm{F}_{2}$ molecule can be seen by examining the errors relative to CCSDT obtained in the CCSD $(\mathrm{T})$ calculations at $R=1.5 R_{e}, 2 R_{e}$, and $5 R_{e}$, which are $-5.711,-23.596$, and -39.348 millihartree, respectively, when the cc-pVDZ basis set is used. As shown in Table II [see the $N_{\operatorname{det}(\text { in })}=1 \mathrm{CC}(P ; Q)$ energies], the CR$\mathrm{CC}(2,3)$ triples correction to CCSD helps, reducing the large errors characterizing CCSD(T) to 1.735 millihartree at $R=1.5 R_{e}, 1.862$ millihartree at $R=2 R_{e}$, and 1.613 millihartree at $R=5 R_{e}$, which are much more acceptable, but, as demonstrated in our earlier active-orbitalbased and semi-stochastic $\operatorname{CC}(P ; Q)$ studies, ${ }^{39,40,42}$ further error reduction requires the relaxation of $T_{1}$ and $T_{2}$ clusters in the presence of the dominant $T_{3}$ contributions. This is what the CIPSI-driven $\operatorname{CC}(P ; Q)$ methodology, where we use CIPSI runs to identify the leading triple excitations for the inclusion in the $P$ space, allows us to do.

Indeed, with as little as 1,006-1,442 $S_{z}=0$ determinants of the $A_{g}\left(D_{2 h}\right)$ symmetry in the final Hamiltonian diagonalization spaces (we used $D_{2 h}$ group, which is the largest Abelian subgroup of the $D_{\infty h}$ symmetry group of $\mathrm{F}_{2}$, in our calculations), generated by the inexpensive $N_{\operatorname{det}(\text { in })}=1,000$ CIPSI runs at $R=1.5 R_{e}, 2 R_{e}$, and $5 R_{e}$, which capture very small fractions, on the order of $0.1-$ $0.2 \%$, of all triples, the errors in the resulting $\mathrm{CC}(P ; Q)$ energies relative to CCSDT are 0.202 millihartree at $R=1.5 R_{e}, 0.132$ millihartree at $R=2 R_{e}$, and 0.144 millihartree at $R=5 R_{e}$. This is an approximately tenfold error reduction compared to the CR-CC $(2,3)$ calcula- tions, in which $T_{1}$ and $T_{2}$ clusters, obtained with CCSD, are decoupled from $T_{3}$, and an improvement of the faulty $\operatorname{CCSD}(\mathrm{T})$ energetics by orders of magnitude. As explained in detail in our papers on the CIQMC/CCMCdriven $\mathrm{CC}(P ; Q)$ approaches, ${ }^{39-41}$ with the fractions of triples in the relevant $P$ spaces being so small, the post-CIPSI steps of the $\operatorname{CC}(P ; Q)$ calculations are not much more expensive than the CCSD-based CR-CC $(2,3)$ computations and a lot faster than the corresponding CCSDT computations. The $\operatorname{CC}(P ; Q)$ calculations using $N_{\operatorname{det}(\text { in) }}=1,000$ do not offer any improvements over CR$\mathrm{CC}(2,3)$ at the equilibrium geometry, since the final diagonalization space of the underlying CIPSI run does not yet contain any triply excited determinants, and the CR$\mathrm{CC}(2,3)$ energy at $R=R_{e}$ is already very accurate anyway, but with the relatively small additional effort corresponding to $N_{\operatorname{det}(\text { in) }}=10,000$, which results in 10,150 $S_{z}=0$ determinants of the $A_{g}\left(D_{2 h}\right)$ symmetry in the final CIPSI diagonalization space and only $1.2 \%$ of all triples in the $P$ space, the unsigned error in the $\mathrm{CC}(P ; Q)$ energy relative to its CCSDT parent substantially decreases, from 0.240 millihartree, when $N_{\operatorname{det}(\text { in })} \leq 1,000$, to 67 microhartree, when $N_{\operatorname{det}(\text { in) }}$ is set at 10,000 . The use of $N_{\operatorname{det}(\text { in) }}=10,000$ for the remaining three geometries considered in Table II and Fig. 1 produces similarly compact $\left|\Psi^{(\mathrm{CIPSI})}\right\rangle$ wave functions, spanned by 11,578 19,957 determinants, similarly small fractions of triples in the corresponding $P$ spaces, ranging from $1.5 \%$ at $R=1.5 R_{e}$ to $2.2 \%$ at $R=5 R_{e}$, and even smaller errors in the $\mathrm{CC}(P ; Q)$ energies relative to CCSDT.

It is clear from Table II and Fig. 1 that the convergence of the CIPSI-driven $\mathrm{CC}(P ; Q)$ energies toward CCSDT with the wave function termination parameter $N_{\operatorname{det}(\text { in) }}$, with the number of determinants used to generate the final CIPSI state $\left|\Psi^{(\mathrm{CIPSI})}\right\rangle\left[N_{\operatorname{det}(\text { out })}\right]$, and with the fraction of triples in the $P$ space captured by the CIPSI procedure is very fast. The uncorrected $\mathrm{CC}(P)$ energies converge to CCSDT too, but they do it at a considerably slower rate than their $\mathrm{CC}(P ; Q)$ counterparts. For example, the CIPSI-driven $\mathrm{CC}(P)$ calculations reduce the $9.485,32.424,45.638$, and 49.816 millihartree errors relative to CCSDT obtained with CCSD to $1.419,0.991,0.922$, and 0.764 millihartree, respectively, when $N_{\operatorname{det}(\text { in) }}=50,000$, which translates in the

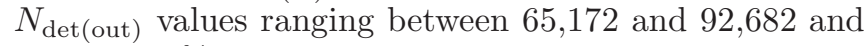
about 5-9\% of all triples included in the underlying $P$ spaces, but the errors characterizing the corresponding $\mathrm{CC}(P ; Q)$ energies are already at the level of $20-30 \mathrm{mi}-$ crohartree at this stage, which is obviously a substantial improvement over the $\mathrm{CC}(P)$ results. It is also worth noticing that the convergence of the CIPSI-driven $\mathrm{CC}(P)$ and $\mathrm{CC}(P ; Q)$ energies toward their CCSDT parents with $N_{\operatorname{det}(\text { in) }}\left[\right.$ or $\left.N_{\operatorname{det}(\text { out })}\right]$ is considerably faster than the convergence of the corresponding variational and perturbatively corrected CIPSI energies toward the extrapolated $E_{\mathrm{var}}+\Delta E_{\mathrm{r}}^{(2)}$ values. This is in line with the above observations that indicate that the CIPSI-driven $\operatorname{CC}(P ; Q)$ calculations are capable of recovering the parent CCSDT 
energetics, even when electronic quasi-degeneracies and $T_{3}$ clusters become significant, out of the unconverged CIPSI runs that rely on relatively small diagonalization spaces. We observed similar patterns when comparing the semi-stochastic, CIQMC- and CCMC-driven, $\mathrm{CC}(P) / \operatorname{EOMCC}(P)$ and $\mathrm{CC}(P ; Q)$ calculations with the underlying CIQMC/CCMC propagations. ${ }^{39-41,56}$

In analogy to the previously considered deterministic, active-orbital-based ${ }^{42,43,45,46,86}$ and semi-stochastic, CIQMC/CCMC-based ${ }^{39,40} \mathrm{CC}(P ; Q)$ studies, the convergence of the CIPSI-driven $\mathrm{CC}(P ; Q)$ computations toward the parent CCSDT energetics remains equally rapid when we use basis sets larger than cc-pVDZ. This is illustrated in Table III, where we show the results of the CIPSI-driven $\mathrm{CC}(P ; Q)$ calculations for the $\mathrm{F}_{2}$ molecule at $R=2 R_{e}$ using the cc-pVTZ basis set. ${ }^{83}$ As pointed out in Ref. 40, and in analogy to the cc-pVDZ basis, the $T_{3}$ contribution characterizing the stretched $\mathrm{F}_{2} / \mathrm{cc}-\mathrm{pVTZ}$ system in which the internuclear separation is set at twice the equilibrium bond length, estimated by forming the difference between the CCSDT and CCSD energies at -62.819 millihartree, is not only very large, but also larger, in absolute value, than the corresponding CCSDT dissociation energy, which is about 57 millihartree, when the CCSDT energy at $R_{e}$ is subtracted from its $5 R_{e}$ counterpart. It is also highly nonperturbative at the same time, as demonstrated by the -26.354 millihartree error relative to CCSDT obtained with $\operatorname{CCSD}(\mathrm{T})$. Again, the CR-CC $(2,3)$ triples correction to CCSD, equivalent to the $N_{\operatorname{det}(\text { in) }}=1 \mathrm{CC}(P ; Q)$ calculation in Table III, works a lot better than $\operatorname{CCSD}(\mathrm{T})$, but the 4.254 millihartree error relative to CCSDT remains. With as little as $5,118 S_{z}=0$ determinants of the $A_{g}\left(D_{2 h}\right)$ symmetry in the final diagonalization space obtained by the nearly effortless $N_{\text {det(in) }}=5,000$ CIPSI run, which captures $0.03 \%$ of all triples, the difference between the $\mathrm{CC}(P ; Q)$ and CCSDT energies decreases to 0.345 millihartree, and with the help of the $N_{\operatorname{det}(i n)}=50,000$ CIPSI calculation, which is still relatively inexpensive, resulting in 82,001 $S_{z}=0 A_{g}\left(D_{2 h}\right)$-symmetric determinants in the final diagonalization space and less than $1 \%$ of the triples in the $P$ space, the error in the $\mathrm{CC}(P ; Q)$ energy relative to its CCSDT parent reduces to less than 0.1 millihartree. Similarly to the cc-pVDZ basis, the convergence of the CIPSI-driven $\mathrm{CC}(P ; Q)$ energies toward CCSDT with $N_{\text {det(in) }}, N_{\text {det(out) }}$, and the fraction of triples in the $P$ space captured by the CIPSI algorithm is not only fast, when the larger cc-pVTZ basis set is employed, but also much faster than in the case of the uncorrected $\mathrm{CC}(P)$ calculations. Once again, as $N_{\text {det(in) }}$ increases, the rate of convergence of the CIPSI-driven $\mathrm{CC}(P)$ and $\mathrm{CC}(P ; Q)$ energies toward their CCSDT parent is higher than those characterizing the corresponding variational and perturbatively corrected CIPSI energies in their attempt to recover the extrapolated $E_{\mathrm{var}}+\Delta E_{\mathrm{r}}^{(2)}$ energy.

Our final test, shown in Table IV, is the frequently examined $39,40,43,87-102$ automerization of cyclobutadiene. In this case, one of the key challenges is an accurate deter- mination of the activation barrier, which requires a wellbalanced description of the nondegenerate, rectangleshaped, closed-shell reactant (or the equivalent product) species, in which electron correlation effects are largely dynamical in nature, and the quasi-degenerate, squareshaped, transition state characterized by substantial nondynamical correlations associated with its strongly diradical character. Experimental estimates of the activation barrier for the automerization of cyclobutadiene, which range from 1.6 to $10 \mathrm{kcal} / \mathrm{mol},{ }^{89,90}$ are not very precise, but the most accurate single- and multi-reference $a b$ initio computations, compiled, for example, in Refs. 43,88 , and 101 , place it in the $6-10 \mathrm{kcal} / \mathrm{mol}$ range. This, in particular, applies to the CCSDT approach, ${ }^{43,87}$ which is of the primary interest in the present study. Indeed, if we, for example, use the reactant and transition-state geometries obtained with the multi-reference averagequadratic CC (MR-AQCC) approach ${ }^{103,104}$ in Ref. 95 and the cc-pVDZ basis set, the CCSDT value of the activation energy characterizing the automerization of cyclobutadiene becomes $7.627 \mathrm{kcal} / \mathrm{mol},{ }^{43}$ in good agreement with the best $a b$ initio calculations carried out to date. By adopting the same geometries and basis set in this initial benchmark study of the CIPSI-driven $\mathrm{CC}(P ; Q)$ methodology, we can examine if the $\mathrm{CC}(P ; Q)$ calculations using the $P$ spaces constructed with the help of CIPSI runs are capable of converging this result. The main challenge here is that the $T_{3}$ effects, estimated as the difference between the CCSDT and CCSD energies, are not only very large, but also hard to balance. When the cc-pVDZ basis set used in this study is employed, they are -26.827 millihartree for the reactant and -47.979 millihartree for the transition state. Furthermore, in the case of the transition state, the coupling of the lower-rank $T_{1}$ and $T_{2}$ clusters with their higherrank $T_{3}$ counterpart is so large that none of the noniterative triples corrections to CCSD provide a reasonable description of the activation barrier. ${ }^{43,87,88}$ This, in particular, applies to the $\mathrm{CR}-\mathrm{CC}(2,3)$ approach, equivalent to the $N_{\operatorname{det}(\text { in })}=1 \mathrm{CC}(P ; Q)$ calculation, which produces an activation barrier exceeding $16 \mathrm{kcal} / \mathrm{mol}$, when the cc-pVDZ basis set is employed, instead of less than 8 kcal/mol obtained with CCSDT (cf. Table IV). The failure of $\mathrm{CR}-\mathrm{CC}(2,3)$ to provide an accurate description of the activation energy is a consequence of its inability to accurately describe the transition state. Indeed, the difference between the $\mathrm{CR}-\mathrm{CC}(2,3)$ and CCSDT energies at the transition-state geometry is 14.636 millihartree, when the cc-pVDZ basis set is employed, as opposed to only 0.848 millihartree obtained for the reactant. As discussed in detail in Refs. 43 and 88, other triples corrections to CCSD, including the widely used CCSD(T) approach, face similar problems. We demonstrated in Refs. 39,40 , and 43 that the deterministic $\mathrm{CC}(P ; Q)$-based $\mathrm{CC}(\mathrm{t} ; 3)$ approach and the semi-stochastic $\mathrm{CC}(P ; Q)$ calculations using CIQMC and CCMC are capable of accurately approximating the CCSDT energies of the reactant and transition-state species and the CCSDT activation 
barrier, so it is interesting to explore if the CIPSI-driven $\mathrm{CC}(P ; Q)$ methodology can do the same.

As shown in Table IV, the $\operatorname{CC}(P ; Q)$ calculations using CIPSI to identify the dominant triply excited determinants for the inclusion in the $P$ space are very efficient in converging the CCSDT energetics. With the final diagonalization spaces spanned by a little over 110,000 $S_{z}=0$ determinants of the $A_{g}\left(D_{2 h}\right)$ symmetry (we used $D_{2 h}$ group for both the $D_{2 h}$-symmetric reactant and the $D_{4 h}$-symmetric transition state in our calculations), generated in the relatively inexpensive CIPSI runs defined by $N_{\operatorname{det}(\text { in })}=100,000$ that capture $0.1 \%$ of all triples, the 0.848 millihartree, 14.636 millihartree, and $8.653 \mathrm{kcal} / \mathrm{mol}$ errors in the reactant, transitionstate, and activation energies relative to CCSDT obtained with CR-CC $(2,3)$ are reduced by factors of $2-$ 4, to 0.382 millihartree, 3.507 millihartree, and 1.961 $\mathrm{kcal} / \mathrm{mol}$, respectively, when the $\operatorname{CC}(P ; Q)$ approach is employed. When $N_{\operatorname{det}(\text { in) }}$ is increased to 500,000 , resulting in about 890,000-900,000 $S_{z}=0$ determinants of the $A_{g}\left(D_{2 h}\right)$ symmetry in the final diagonalization spaces used by CIPSI and 1.0-1.2\% of the triples in the resulting $P$ spaces, the errors in the $\operatorname{CC}(P ; Q)$ reactant, transition-state, and activation energies relative to CCSDT become 0.267 millihartree, 0.432 millihartree, and $0.104 \mathrm{kcal} / \mathrm{mol}$. Clearly, these are great improvements compared to the initial $N_{\operatorname{det}(\text { in) }}=1$, i.e., CR$\mathrm{CC}(2,3)$, values, especially if we realize that with the fractions of triples being so small, the post-CIPSI steps of the $\operatorname{CC}(P ; Q)$ computations are not only a lot faster than the parent CCSDT runs, but also not much more expensive than the corresponding $\mathrm{CR}-\mathrm{CC}(2,3)$ calculations, as elaborated on in Refs. 39-41.

In analogy to the previously discussed case of bond breaking in $\mathrm{F}_{2}$, the convergence of the CIPSI-driven $\mathrm{CC}(P ; Q)$ energies toward CCSDT for the reactant and transition-state species defining the automerization of cyclobutadiene with $N_{\operatorname{det}(\text { in) }}, N_{\operatorname{det}(\text { out) }}$, and the fractions of triples in the relevant $P$ spaces captured by the underlying CIPSI runs is not only very fast, but also significantly faster than that characterizing the uncorrected $\mathrm{CC}(P)$ calculations. For each of the two species, the $\mathrm{CC}(P)$ energies converge toward their CCSDT parent in a steady fashion, but, as shown in Table IV, their convergence is rather slow, emphasizing the importance of correcting the results of the $\mathrm{CC}(P)$ calculations for the missing triple excitations not captured by the CIPSI runs using smaller diagonalization spaces. Similarly to the previously examined active-orbital-based ${ }^{42,43,45,46,86}$ and CIQMC/CCMC-based ${ }^{39,40} \mathrm{CC}(P ; Q)$ approaches, our moment correction $\delta(P ; Q)$, defined by Eq. (9), is very effective in this regard. Another interesting observation, which can be made based on the results presented in Table IV, is that while the $\mathrm{CC}(P)$ energies for the individual reactant and transition-state species converge toward their CCSDT parent values in a steady fashion, the corresponding activation barrier values behave in a less systematic manner, oscillating between about -1 and
$1 \mathrm{kcal} / \mathrm{mol}$ when $N_{\operatorname{det}(\mathrm{in})}=500,000-15,000,000$. One might try to eliminate this behavior, which is a consequence of a different character of the many-electron correlation effects in the reactant and transition-state species, by merging the $P$ spaces used to perform the $\mathrm{CC}(P)$ calculations for the two structures, but, as shown in Table IV, the $\operatorname{CC}(P ; Q)$ correction $\delta(P ; Q)$, which is highly effective in capturing the missing $T_{3}$ correlations, takes care of this problem too. As $N_{\operatorname{det}(\text { in) }}, N_{\operatorname{det}(\text { out })}$, and the fractions of triples in the $P$ spaces used in the $\mathrm{CC}(P)$ calculations for the reactant and transition state increase, the $\mathrm{CC}(P ; Q)$ values of the activation barrier converge toward its CCSDT parent rapidly and in a smooth manner, eliminating, at least to a large extent, the need to equalize the $P$ spaces used in the underlying $\mathrm{CC}(P)$ steps. As in the case of bond breaking in the fluorine molecule, the convergence of the CIPSI-driven $\mathrm{CC}(P)$ and $\mathrm{CC}(P ; Q)$ energies toward their CCSDT parents with $N_{\operatorname{det}(\text { in) }} / N_{\operatorname{det}(\text { out })}$ is considerably faster than the convergence of the variational and perturbatively corrected CIPSI energies toward the extrapolated $E_{\mathrm{var}}+\Delta E_{\mathrm{r}}^{(2)}$ values, but we must keep in mind that the calculated CCSDT and extrapolated $E_{\mathrm{var}}+\Delta E_{\mathrm{r}}^{(2)}$ energies, while representing the respective parent limits for the $\operatorname{CC}(P ; Q)$ and CIPSI calculations, are fundamentally different quantities, especially when higher-than-triply excited cluster components, which are not considered in this work, become significant. As one might anticipate, the $N_{\operatorname{det}(\text { in) }}$ values needed to accurately represent the CCSDT energies of the reactant and transition-state species of cyclobutadiene by the CIPSI-driven $\operatorname{CC}(P ; Q)$ approach are considerably larger than those used in the analogous $\mathrm{CC}(P ; Q)$ calculations for the smaller $\mathrm{F}_{2}$ system, but they are orders of magnitude smaller than the values of $N_{\operatorname{det}(\text { in) }}$ required to obtain the similarly well converged $E_{\mathrm{var}}+\Delta E_{\mathrm{r}}^{(2)}$ energetics in the underlying CIPSI runs.

\section{CONCLUSIONS}

Inspired by our recent studies ${ }^{39-41}$ aimed at determining accurate electronic energies equivalent to the results of high-level CC calculations, in which we combined the deterministic $\mathrm{CC}(P ; Q)$ framework ${ }^{42-46}$ with the stochastic CIQMC ${ }^{47-51}$ and $\mathrm{CCMC}^{52-55}$ propagations, and the successes of modern formulations ${ }^{61-71}$ of selected CI techniques, ${ }^{57-60}$ we have proposed a new form of the $\operatorname{CC}(P ; Q)$ approach, in which we identify the dominant higher-than-doubly excited determinants for the inclusion in the underlying $P$ spaces with the help of the selected CI algorithm abbreviated as CIPSI. ${ }^{59,70,71}$ To illustrate potential benefits offered by the proposed merger of the $\mathrm{CC}(P ; Q)$ and CIPSI methodologies, we have implemented the CIPSI-driven $\operatorname{CC}(P ; Q)$ method designed to converge CCSDT energetics. The advantages of the CIPSI-driven $\operatorname{CC}(P ; Q)$ methodology have been il- 
lustrated by a few numerical examples, including bond breaking in $\mathrm{F}_{2}$ and the automerization of cyclobutadiene, which are accurately described by CCSDT.

The reported benchmark calculations demonstrate that the convergence of the CIPSI-driven $\mathrm{CC}(P ; Q)$ energies toward CCSDT with the wave function termination parameter $N_{\operatorname{det}(i n)}$ adopted by CIPSI, with the number of determinants used to generate the final CIPSI state $\left[N_{\operatorname{det}(\text { out })}\right]$, and with the fractions of triples in the $P$ space captured by the CIPSI procedure is very fast. As a result, one can obtain CCSDT-level energetics, even when electronic quasi-degeneracies and $T_{3}$ clusters become substantial, based on the information extracted from the relatively inexpensive CIPSI runs. This can be attributed to two key factors. The first one is a tempered wave function growth through iterative Hamiltonian diagonalizations in the modern implementation of CIPSI available in Quantum Package 2.0, ${ }^{70,71}$ which we utilized in this work, resulting in an economical selection of the dominant triply excited determinants (in general, the dominant higher-than-doubly excited determinants) for the inclusion in the $P$ spaces driving the $\mathrm{CC}(P ; Q)$ computations. The second one is the efficiency of the moment corrections $\delta(P ; Q)$ defining the $\mathrm{CC}(P ; Q)$ formalism, which provide an accurate and robust description of the missing $T_{3}$ contributions that cannot be captured by the underlying $\mathrm{CC}(P)$ calculations using small fractions of triples identified by the CIPSI runs employing smaller diagonalization spaces. We have also shown that the uncorrected $\mathrm{CC}(P)$ energies converge with $N_{\operatorname{det}(\text { in) }}, N_{\operatorname{det}(\text { out) }}$, and the fractions of triples in the $P$ spaces constructed with the help of CIPSI to their CCSDT parent values too, but they do it at a much slower rate, so that we do not recommend the uncorrected $\mathrm{CC}(P)$ approach in calculations aimed at recovering high-level CC energetics.

Clearly, the present study is only our initial exploration of the CIPSI-driven (or, in general, selectedCI-driven) $\mathrm{CC}(P ; Q)$ methodology, which needs more work. In addition to code optimization and more numerical tests, especially including larger molecules and basis sets, we would like to extend the proposed CIPSIdriven $\mathrm{CC}(P ; Q)$ framework to higher levels of the $\mathrm{CC}$ theory, beyond CCSDT, as we already did in the context of the active-orbital-based ${ }^{45,46}$ and CIQMC-based ${ }^{40}$ $\mathrm{CC}(P ; Q)$ considerations, and examine if other selected CI methods, such as heat-bath $\mathrm{CI}^{67-69}$ or adaptive$\mathrm{CI},{ }^{61,62}$ to mention a couple of examples, are as useful in the context of $\mathrm{CC}(P ; Q)$ considerations as the CIPSI approach adopted in this work. Following our recent semi-stochastic $\operatorname{EOMCC}(P)$ and $\mathrm{CC}(P ; Q)$ work, ${ }^{41,56}$ we are also planning to extend the CIPSI-driven $\mathrm{CC}(P ; Q)$ methodology to excited electronic states. One of the main advantages of CIPSI and other selected-CI methods, which are based on Hamiltonian diagonalizations, is that they can be easily applied to excited states (see, e.g., Refs. 62, 105-110). This would allow us to construct the state-specific $P$ spaces, adjusted to the individual electronic states of interest, which is more difficult to accomplish within the CIQMC framework (see Refs. 41 and 56 for additional comments). Encouraged by our recent work on the semi-stochastic $\mathrm{CC}(P ; Q)$ models using truncated CIQMC rather than FCIQMC propagations to determine the underlying $P$ spaces,${ }^{40}$ we would like to examine if one can replace the unconstrained CIPSI algorithm used in this study, which explores the entire many-electron Hilbert space in the iterative wave function build-up, by its less expensive truncated analogs compatible with the determinantal spaces needed in the CC calculations of interest (e.g., the CISDT or CISDTQ analogs of CIPSI if one is interested in converging the CCSDT or CCSDTQ energetics through CIPSIdriven $\mathrm{CC}(P ; Q)$ computations). This may help us to achieve the desired high accuracy levels in the CIPSIdriven $\mathrm{CC}(P ; Q)$ calculations with the relatively short CI wave function expansions, even when larger systems are examined, since the diagonalization spaces generated by the truncated CIPSI models will be significantly smaller than those produced when CIPSI is allowed to explore the entire many-electron Hilbert space. Last, but not least, inspired by our recent work on the CIPSI-driven externally corrected CC models, ${ }^{111}$ we would like to investigate the effect of the CIPSI input parameter $f$ that controls the wave function growth in successive Hamiltonian diagonalizations, which was set in this study at the default value of 2 , on the rate of convergence of the CIPSI-driven $\mathrm{CC}(P ; Q)$ energies toward their high-level CC parents, such as those obtained with CCSDT.

\section{ACKNOWLEDGMENTS}

This work has been supported by the Chemical Sciences, Geosciences and Biosciences Division, Office of Basic Energy Sciences, Office of Science, U.S. Department of Energy (Grant No. DE-FG02-01ER15228 to P.P), and Phase I and II Software Fellowships awarded to J.E.D. by the Molecular Sciences Software Institute funded by the National Science Foundation grant ACI-1547580. We thank Drs. Pierre-François Loos and Anthony Scemama for useful discussions about Quantum Package 2.0 employed in our CIPSI computations.

\section{DATA AVAILABILITY}

The data that support the findings of this study are available within the article.

\section{REFERENCES}

\footnotetext{
${ }^{1}$ J. Hubbard, Proc. R. Soc. Lond., Ser. A 240, 539 (1957).

${ }^{2}$ N. M. Hugenholtz, Physica 23, 481 (1957).

${ }^{3}$ F. Coester, Nucl. Phys. 7, 421 (1958).

${ }^{4}$ F. Coester and H. Kümmel, Nucl. Phys. 17, 477 (1960).

${ }^{5}$ J. Č́́žek, J. Chem. Phys. 45, 4256 (1966).
} 
${ }^{6}$ J. Č́ížek, Adv. Chem. Phys. 14, 35 (1969).

${ }^{7}$ J. Paldus, J. Č́ižek, and I. Shavitt, Phys. Rev. A 5, 50 (1972).

${ }^{8}$ J. Paldus and X. Li, Adv. Chem. Phys. 110, 1 (1999).

${ }^{9}$ R. J. Bartlett and M. Musial, Rev. Mod. Phys. 79, 291 (2007).

${ }^{10}$ G. D. Purvis, III and R. J. Bartlett, J. Chem. Phys. 76, 1910 (1982).

${ }^{11}$ J. M. Cullen and M. C. Zerner, J. Chem. Phys. 77, 4088 (1982).

${ }^{12}$ J. Noga and R. J. Bartlett, J. Chem. Phys. 86, 7041 (1987), 89, 3401 (1988) [Erratum].

${ }^{13}$ G. E. Scuseria and H. F. Schaefer, III, Chem. Phys. Lett. 152, 382 (1988).

14 J. D. Watts and R. J. Bartlett, J. Chem. Phys. 93, 6104 (1990).

${ }^{15}$ N. Oliphant and L. Adamowicz, J. Chem. Phys. 95, 6645 (1991).

${ }^{16}$ S. A. Kucharski and R. J. Bartlett, J. Chem. Phys. 97, 4282 (1992).

${ }^{17}$ P. Piecuch and L. Adamowicz, J. Chem. Phys. 100, 5792 (1994).

${ }^{18}$ K. Emrich, Nucl. Phys. A 351, 379 (1981).

${ }^{19}$ J. Geertsen, M. Rittby, and R. J. Bartlett, Chem. Phys. Lett. 164, 57 (1989).

${ }^{20}$ J. F. Stanton and R. J. Bartlett, J. Chem. Phys. 98, 7029 (1993).

${ }^{21}$ K. Kowalski and P. Piecuch, J. Chem. Phys. 115, 643 (2001).

${ }^{22}$ K. Kowalski and P. Piecuch, Chem. Phys. Lett. 347, 237 (2001).

${ }^{23}$ S. A. Kucharski, M. Włoch, M. Musiał, and R. J. Bartlett, J. Chem. Phys. 115, 8263 (2001).

${ }^{24}$ M. Kállay and J. Gauss, J. Chem. Phys. 121, 9257 (2004).

${ }^{25}$ S. Hirata, J. Chem. Phys. 121, 51 (2004).

${ }^{26}$ H. Monkhorst, Int. J. Quantum Chem. Symp. 11, 421 (1977).

${ }^{27}$ E. Dalgaard and H. Monkhorst, Phys. Rev. A 28, 1217 (1983).

${ }^{28}$ D. Mukherjee and P. K. Mukherjee, Chem. Phys. 39, 325 (1979).

${ }^{29}$ H. Sekino and R. J. Bartlett, Int. J. Quantum Chem. Symp. 18, 255 (1984).

${ }^{30}$ M. Takahashi and J. Paldus, J. Chem. Phys. 85, 1486 (1986).

${ }^{31}$ H. Koch and P. Jørgensen, J. Chem. Phys. 93, 3333 (1990).

${ }^{32}$ H. Koch, H. J. A. Jensen, P. Jørgensen, and T. Helgaker, J. Chem. Phys. 93, 3345 (1990).

${ }^{33}$ A. E. Kondo, P. Piecuch, and J. Paldus, J. Chem. Phys. 102, 6511 (1995).

${ }^{34}$ A. E. Kondo, P. Piecuch, and J. Paldus, J. Chem. Phys. 104, 8566 (1995).

${ }^{35}$ K. Raghavachari, G. W. Trucks, J. A. Pople, and M. HeadGordon, Chem. Phys. Lett. 157, 479 (1989).

${ }^{36}$ P. Piecuch, K. Kowalski, I. S. O. Pimienta, and M. J. McGuire, Int. Rev. Phys. Chem. 21, 527 (2002).

${ }^{37}$ P. Piecuch, K. Kowalski, I. S. O. Pimienta, P.-D. Fan, M. Lodriguito, M. J. McGuire, S. A. Kucharski, T. Kuś, and M. Musiał, Theor. Chem. Acc. 112, 349 (2004).

${ }^{38}$ P. Piecuch, Mol. Phys. 108, 2987 (2010).

${ }^{39}$ J. E. Deustua, J. Shen, and P. Piecuch, Phys. Rev. Lett. 119, 223003 (2017)

${ }^{40}$ J. E. Deustua, J. Shen, and P. Piecuch, J. Chem. Phys. 154, 124103 (2021)

${ }^{41}$ S. H. Yuwono, A. Chakraborty, J. E. Deustua, J. Shen, and P. Piecuch, Mol. Phys. 118, e1817592 (2020)

${ }^{42}$ J. Shen and P. Piecuch, Chem. Phys. 401, 180 (2012).

${ }^{43}$ J. Shen and P. Piecuch, J. Chem. Phys. 136, 144104 (2012).

${ }^{44}$ J. Shen and P. Piecuch, J. Chem. Theory Comput. 8, 4968 (2012).

${ }^{45}$ N. P. Bauman, J. Shen, and P. Piecuch, Mol. Phys. 115, 2860 (2017).

${ }^{46}$ I. Magoulas, N. P. Bauman, J. Shen, and P. Piecuch, J. Phys. Chem. A 122, 1350 (2018).

${ }^{47}$ G. H. Booth, A. J. W. Thom, and A. Alavi, J. Chem. Phys. 131, 054106 (2009).
${ }^{48}$ D. Cleland, G. H. Booth, and A. Alavi, J. Chem. Phys. 132, 041103 (2010).

${ }^{49}$ W. Dobrautz, S. D. Smart, and A. Alavi, J. Chem. Phys. 151, 094104 (2019).

${ }^{50}$ K. Ghanem, A. Y. Lozovoi, and A. Alavi, J. Chem. Phys. 151, 224108 (2019).

${ }^{51}$ K. Ghanem, K. Guther, and A. Alavi, J. Chem. Phys. 153, 224115 (2020)

${ }^{52}$ A. J. W. Thom, Phys. Rev. Lett. 105, 263004 (2010).

${ }^{53}$ R. S. T. Franklin, J. S. Spencer, A. Zoccante, and A. J. W. Thom, J. Chem. Phys. 144, 044111 (2016).

${ }^{54}$ J. S. Spencer and A. J. W. Thom, J. Chem. Phys. 144, 084108 (2016).

${ }^{55}$ C. J. C. Scott and A. J. W. Thom, J. Chem. Phys. 147, 124105 (2017).

${ }^{56}$ J. E. Deustua, S. H. Yuwono, J. Shen, and P. Piecuch, J. Chem. Phys. 150, 111101 (2019).

${ }^{57}$ J. Whitten and M. Hackmeyer, J. Chem. Phys. 51, 5584 (1969).

${ }^{58}$ C. Bender and E. Davidson, Phys. Rev. 183, 23 (1969).

${ }^{59}$ B. Huron, J. P. Malrieu, and P. Rancurel, J. Chem. Phys. 58, 5745 (1973).

${ }^{60}$ R. Buenker and S. Peyerimhoff, Theor. Chim. Acta. 35, 33 (1974).

${ }^{61}$ J. Schriber and F. Evangelista, J. Chem. Phys. 144, 161106 (2016).

${ }^{62}$ J. Schriber and F. Evangelista, J. Chem. Theory Comput. 13, 5354 (2017).

${ }^{63}$ N. M. Tubman, J. Lee, T. Takeshita, M. Head-Gordon, and K. Whaley, J. Chem. Phys. 145, 044112 (2016).

${ }^{64}$ N. M. Tubman, C. Freeman, D. Levine, D. Hait, M. HeadGordon, and K. Whaley, J. Chem. Theory Comput. 16, 2139 (2020).

${ }^{65}$ W. Liu and M. Hoffmann, J. Chem. Theory Comput. 12, 1169 (2016), 12, 3000 (2016) [Erratum]

${ }^{66}$ N. Zhang, W. Liu, and M. Hoffmann, J. Chem. Theory Comput. 16, 2296 (2020)

${ }^{67}$ A. A. Holmes, N. M. Tubman, and C. J. Umrigar, J. Chem. Theory Comput. 12, 3674 (2016).

${ }^{68}$ S. Sharma, A. A. Holmes, G. Jeanmairet, A. Alavi, and C. J. Umrigar, J. Chem. Theory Comput. 13, 1595 (2017).

${ }^{69} \mathrm{~J}$. Li, M. Otten, A. A. Holmes, S. Sharma, and C. J. Umrigar, J. Chem. Phys. 149, 214110 (2018).

${ }^{70}$ Y. Garniron, A. Scemama, P.-F. Loos, and M. Caffarel, J. Chem. Phys. 147, 034101 (2017).

${ }^{71}$ Y. Garniron, T. Applencourt, K. Gasperich, A. Benali, A. Ferte, J. Paquier, B. Pradines, R. Assaraf, P. Reinhardt, J. Toulouse, P. Barbaresco, N. Renon, G. David, J.-P. Malrieu, M. Veril, M. Caffarel, P.-F. Loos, E. Giner, and A. Scemama, J. Chem. Theory Comput. 15, 3591 (2019).

${ }^{72}$ P.-F. Loos, Y. Damour, and A. Scemama, J. Chem. Phys. 153, 176101 (2020)

${ }^{73}$ J. J. Eriksen, T. A. Anderson, J. E. Deustua, K. Ghanem, D. Hait, M. R. Hoffmann, S. Lee, D. S. Levine, I. Magoulas, J. Shen, N. M. Tubman, K. B. Whaley, E. Xu, Y. Yao, N. Zhang, A. Alavi, G. K.-L. Chan, M. Head-Gordon, W. Liu, P. Piecuch, S. Sharma, S. L. Ten-no, C. J. Umrigar, and J. Gauss, J. Phys. Chem. Lett. 11, 8922 (2020).

${ }^{74}$ K. Jankowski, J. Paldus, and P. Piecuch, Theor. Chim. Acta 80, 223 (1991).

${ }^{75}$ P. Piecuch and K. Kowalski, in Computational Chemistry: Reviews of Current Trends, Vol. 5, edited by J. Leszczyński (World Scientific, Singapore, 2000) pp. 1-104.

${ }^{76}$ K. Kowalski and P. Piecuch, J. Chem. Phys. 113, 18 (2000).

${ }^{77}$ P. Piecuch and M. Włoch, J. Chem. Phys. 123, 224105 (2005). 
${ }^{78}$ P. Piecuch, M. Włoch, J. R. Gour, and A. Kinal, Chem. Phys. Lett. 418, 467 (2006).

${ }^{79}$ M. Włoch, M. D. Lodriguito, P. Piecuch, and J. R. Gour, Mol. Phys. 104, 2149 (2006), 104, 2991 (2006) [Erratum].

${ }^{80}$ M. Włoch, J. R. Gour, and P. Piecuch, J. Phys. Chem. A 111, 11359 (2007).

${ }^{81}$ M. W. Schmidt, K. K. Baldridge, J. A. Boatz, S. T. Elbert, M. S. Gordon, J. H. Jensen, S. Koseki, N. Matsunaga, K. A. Nguyen, S. J. Su, T. L. Windus, M. Dupuis, and J. A. Montgomery, J. Comput. Chem. 14, 1347 (1993).

${ }^{82}$ G. M. J. Barca, C. Bertoni, L. Carrington, D. Datta, N. De Silva, J. E. Deustua, D. G. Fedorov, J. R. Gour, A. O. Gunina, E. Guidez, T. Harville, S. Irle, J. Ivanic, K. Kowalski, S. S. Leang, H. Li, W. Li, J. J. Lutz, I. Magoulas, J. Mato, V. Mironov, H. Nakata, B. Q. Pham, P. Piecuch, D. Poole, S. R. Pruitt, A. P. Rendell, L. B. Roskop, K. Ruedenberg, T. Sattasathuchana, M. W. Schmidt, J. Shen, L. Slipchenko, M. Sosonkina, V. Sundriyal, A. Tiwari, J. L. G. Vallejo, B. Westheimer, M. Włoch, P. Xu, F. Zahariev, and M. S. Gordon, J. Chem. Phys. 152, 154102 (2020).

${ }^{83}$ T. H. Dunning, Jr., J. Chem. Phys. 90, 1007 (1989).

${ }^{84}$ K. Kowalski and P. Piecuch, Chem. Phys. Lett. 344, 165 (2001).

${ }^{85}$ M. Musiał and R. J. Bartlett, J. Chem. Phys. 122, 224102 (2005).

${ }^{86}$ S. H. Yuwono, I. Magoulas, J. Shen, and P. Piecuch, Mol. Phys. 117, 1486 (2019).

${ }^{87}$ A. Balková and R. J. Bartlett, J. Chem. Phys. 101, 8972 (1994).

${ }^{88}$ D. I. Lyakh, V. F. Lotrich, and R. J. Bartlett, Chem. Phys. Lett. 501, 166 (2011).

${ }^{89}$ D. W. Whitman and B. K. Carpenter, J. Am. Chem. Soc. 104, 6473 (1982).

${ }^{90}$ B. K. Carpenter, J. Am. Chem. Soc. 105, 1700 (1983).

${ }^{91}$ B. A. Hess, P. Čarsky, and L. J. Schaad, J. Am. Chem. Soc. 105, 695 (1983).

${ }^{92}$ A. F. Voter and W. A. Goddard III, J. Am. Chem. Soc. 108, 2830 (1986).

${ }^{93}$ P. Čarsky, R. J. Bartlett, G. Fitzgerald, J. Nova, and V. Špirko, J. Chem. Phys. 89, 3008 (1988).

${ }^{94}$ O. Demel and J. Pittner, J. Chem. Phys. 124, 144112 (2006).

${ }^{95}$ M. Eckert-Maksić, M. Vazdar, M. Barbatti, H. Lischka, and Z. B. Maksić, J. Chem. Phys. 125, 064310 (2006).

${ }^{96}$ K. Bhaskaran-Nair, O. Demel, and J. Pittner, J. Chem. Phys. 129, 184105 (2008).

${ }^{97}$ P. B. Karadakov, J. Phys. Chem. A 112, 7303 (2008).

${ }^{98}$ O. Demel, K. R. Shamasundar, L. Kong, and M. Nooijen, J. Phys. Chem. A 112, 11895 (2008).

${ }^{99}$ J. Shen, T. Fang, S. Li, and Y. Jiang, J. Phys. Chem. A 112, 12518 (2008).

${ }^{100}$ X. Li and J. Paldus, J. Chem. Phys. 131, 114103 (2009).

${ }^{101}$ T. Zhang, C. Li, and F. A. Evangelista, J. Chem. Theory Comput. 15, 4399 (2019).

${ }^{102}$ G. J. R. Aroeira, M. M. Davis, J. M. Turney, and H. F. Schaefer, J. Chem. Theory Comput. 17, 182 (2021).

${ }^{103}$ P. G. Szalay and R. J. Bartlett, Chem. Phys. Lett. 214, 481 (1993).

${ }^{104}$ P. G. Szalay and R. J. Bartlett, J. Chem. Phys. 103, 3600 (1995).

${ }^{105}$ A. D. Chien, A. A. Holmes, M. Otten, C. J. Umrigar, S. Sharma, and P. M. Zimmerman, J. Phys. Chem. A 122, 2714 (2018).

${ }^{106}$ P.-F. Loos, A. Scemama, A. Blondel, Y. Garniron, M. Caffarel, and D. Jacquemin, J. Chem. Theory Comput. 14, 4360 (2018).

${ }^{107}$ P.-F. Loos, M. Boggio-Pasqua, A. Scemama, M. Caffarel, and D. Jacquemin, J. Chem. Theory Comput. 15, 1939 (2019).
${ }^{108}$ P.-F. Loos, F. Lipparini, M. Boggio-Pasqua, A. Scemama, and D. Jacquemin, J. Chem. Theory Comput. 16, 1711 (2020).

${ }^{109}$ P.-F. Loos, A. Scemama, and D. Jacquemin, J. Phys. Chem. Lett. 11, 2374 (2020).

${ }^{110}$ P.-F. Loos, A. Scemama, M. Boggio-Pasqua, and D. Jacquemin, J. Chem. Theory Comput. 16, 3720 (2020).

${ }^{111}$ I. Magoulas, K. Gururangan, P. Piecuch, J. E. Deustua, and J. Shen, J. Chem. Theory Comput. 17, 4006 (2021). 
TABLE I. Numerical demonstration of the size extensivity of the CIPSI-driven $\mathrm{CC}(P)$ and $\mathrm{CC}(P ; Q)$ approaches, alongside the analogous CCSD, CR-CC $(2,3)$, and CCSDT calculations, using the noninteracting $\mathrm{F}_{2}+\mathrm{Ne}$ system, as described by the cc-pVDZ basis set, in which the $\mathrm{F}-\mathrm{F}$ bond length $R$ was fixed at twice its equilibrium value. In all post-RHF calculations, the core orbitals correlating with the $1 s$ shells of the fluorine and neon atoms were frozen and the Cartesian components of $d$ orbitals were employed throughout. All energy values are total electronic energies in hartree.

\begin{tabular}{|c|c|c|c|c|}
\hline Method & $E\left(\mathrm{~F}_{2}+\mathrm{Ne}\right)^{\mathrm{a}}$ & $E\left(\mathrm{~F}_{2}\right)^{\mathrm{b}}$ & $E(\mathrm{Ne})$ & $E\left(\mathrm{~F}_{2}+\mathrm{Ne}\right)-\left[E\left(\mathrm{~F}_{2}\right)+E(\mathrm{Ne})\right]$ \\
\hline $\mathrm{CCSD}^{\mathrm{C}}$ & -327.692849962 & -199.012562571 & -128.680287394 & 0.000000003 \\
\hline CR-CC $(2,3)^{\mathrm{d}}$ & -327.737915219 & -199.056339293 & -128.681575920 & -0.000000006 \\
\hline $\mathrm{CC}(P) / N_{\operatorname{det}(\text { in })}=5,000$ & $-327.736961010^{\mathrm{e}}$ & $-199.056233029^{\mathrm{f}}$ & $-128.680728060^{\mathrm{g}}$ & 0.000000080 \\
\hline $\mathrm{CC}(P ; Q) / N_{\operatorname{det}(\mathrm{in})}=5,000$ & $-327.739651938^{\mathrm{e}}$ & $-199.058190353^{f}$ & $-128.681461627^{g}$ & 0.000000040 \\
\hline CCSDT & -327.739605196 & -199.058201287 & -128.681403900 & -0.000000009 \\
\hline
\end{tabular}

a The noninteracting $\mathrm{F}_{2}+\mathrm{Ne}$ system was obtained by placing the Ne atom along the axis of the $\mathrm{F}-\mathrm{F}$ bond at 1,000 bohr from the center of mass of the stretched fluorine molecule in which the internuclear separation $R$ was set at $2 R_{e}$, where $R_{e}=2.66816$ bohr is the equilibrium geometry of $\mathrm{F}_{2}$.

b The stretched $\mathrm{F}_{2}$ molecule in which the $\mathrm{F}-\mathrm{F}$ bond length $R$ was set at $2 R_{e}$.

c Equivalent to the $\mathrm{CC}(P)$ calculations with $N_{\operatorname{det}(\text { in })}=1$.

d Equivalent to the $\mathrm{CC}(P ; Q)$ calculations with $N_{\operatorname{det}(\text { in })}=1$.

e The $P$ space used in the $\mathrm{CC}(P)$ calculation for the $\mathrm{F}_{2}+$ Ne system consisted of all singles and doubles and a subset of triples contained in the final $\left|\Psi^{(\mathrm{CIPSI})}\right\rangle$ state of the underlying $N_{\operatorname{det}(\mathrm{in})}=5,000 \mathrm{CIPSI}$ run. The $Q$ space needed to compute the CC(P;Q) correction $\delta(P ; Q)$ was defined as the remaining triples absent in $\left|\Psi^{(\mathrm{CIPSI})}\right\rangle$. The $N_{\operatorname{det}(\mathrm{in})}=5,000 \mathrm{CIPSI}$ run for $\mathrm{F}_{2}+\mathrm{Ne}$, which was initiated from the RHF reference determinant, used $f=2$ and $\eta=10^{-6}$ hartree.

f The $P$ space used in the $\mathrm{CC}(P)$ calculation for $\mathrm{F}_{2}$ was obtained by removing the triply excited determinants involving Ne orbitals from the list of triples provided by the $N_{\operatorname{det}(\text { in })}=5,000$ CIPSI run for the $\mathrm{F}_{2}+$ Ne system. The $Q$ space needed to compute the corresponding $\operatorname{CC}(P ; Q)$ correction $\delta(P ; Q)$ was defined as the remaining triples missing in the $P$ space.

$\mathrm{g}$ The $P$ space used in the $\mathrm{CC}(P)$ calculation for Ne was obtained by removing the triply excited determinants involving $\mathrm{F}_{2}$ orbitals from the list of triples provided by the $N_{\operatorname{det}(\text { in })}=5,000$ CIPSI run for the $\mathrm{F}_{2}+$ Ne system. The $Q$ space needed to compute the corresponding $\mathrm{CC}(P ; Q)$ correction $\delta(P ; Q)$ was defined as the remaining triples missing in the $P$ space. 
TABLE II. Convergence of the $\mathrm{CC}(P)$ and $\mathrm{CC}(P ; Q)$ energies toward CCSDT, alongside the variational and perturbatively corrected CIPSI energies, for the $\mathrm{F}_{2} / \mathrm{cc}-\mathrm{pVDZ}$ molecule in which the $\mathrm{F}-\mathrm{F}$ bond length $R$ was set at $R_{e}, 1.5 R_{e}, 2 R_{e}$, and $5 R_{e}$, where $R_{e}=2.66816$ bohr is the equilibrium geometry. For each value of the wave function termination parameter $N_{\text {det(in) }}$, the $P$ space used in the $\mathrm{CC}(P)$ calculations consisted of all singles and doubles and a subset of triples contained in the final $\left|\Psi^{(\mathrm{CIPSI})}\right\rangle$ state of the underlying CIPSI run, whereas the $Q$ space needed to compute the corresponding $\mathrm{CC}(P ; Q)$ correction $\delta(P ; Q)$ was defined as the remaining triples absent in $\left|\Psi^{(\mathrm{CIPSI})}\right\rangle$. In all post-RHF calculations, the two lowest-lying core orbitals were frozen and the Cartesian components of $d$ orbitals were employed throughout. Each CIPSI run was initiated from the RHF reference determinant and the MBPT-based stopping parameter $\eta$ was set at $10^{-6}$ hartree. The input parameter $f$ controlling the CIPSI wave function growth was set at the default value of 2 .

\begin{tabular}{|c|c|c|c|c|c|c|c|}
\hline$R / R_{e}$ & $N_{\operatorname{det}(\text { in })} / N_{\operatorname{det}(\text { out })}$ & $\%$ of triples & $E_{\mathrm{var}}{ }^{\mathrm{a}}$ & $E_{\mathrm{var}}+\Delta E^{(2) \mathrm{a}}$ & $E_{\mathrm{var}}+\Delta E_{\mathrm{r}}^{(2) \mathrm{a}}$ & $\mathrm{CC}(P)^{\mathrm{b}}$ & $\mathrm{CC}(P ; Q)^{\mathrm{b}}$ \\
\hline \multirow[t]{11}{*}{1.0} & $1 / 1$ & 0 & $418.057^{\mathrm{C}}$ & $-94.150^{\mathrm{d}}$ & -12.651 & $9.485^{\mathrm{e}}$ & $-0.240^{\mathrm{f}}$ \\
\hline & $10 / 17$ & 0 & 330.754 & -32.707 & -4.877 & 9.485 & -0.240 \\
\hline & $100 / 154$ & 0 & 232.186 & -7.963 & 2.338 & 9.485 & -0.240 \\
\hline & $1,000 / 1,266$ & 0 & 65.926 & 1.480 & 2.079 & 9.485 & -0.240 \\
\hline & $5,000 / 5,072$ & 0.4 & 23.596 & $-0.133(0)$ & $-0.069(0)$ & 4.031 & -0.129 \\
\hline & $10,000 / 10,150$ & 1.2 & 19.197 & $0.045(2)$ & $0.084(2)$ & 3.010 & -0.067 \\
\hline & $50,000 / 81,288$ & 7.9 & 11.282 & $0.133(1)$ & $0.145(1)$ & 1.419 & -0.031 \\
\hline & $100,000 / 162,430$ & 14.5 & 9.222 & $0.138(1)$ & $0.146(1)$ & 0.983 & -0.020 \\
\hline & $500,000 / 649,849$ & 34.3 & 5.630 & $0.092(1)$ & $0.095(1)$ & 0.519 & -0.009 \\
\hline & $1,000,000 / 1,300,305$ & 42.2 & 4.816 & $0.072(0)$ & $0.074(0)$ & 0.464 & -0.008 \\
\hline & $5,000,000 / 5,187,150$ & 85.1 & 1.161 & $0.015(2)$ & $0.016(2)$ & 0.023 & -0.001 \\
\hline \multirow[t]{11}{*}{1.5} & $1 / 1$ & 0 & $541.109^{\mathrm{c}}$ & $-130.718^{\mathrm{d}}$ & 137.819 & $32.424^{\mathrm{e}}$ & $1.735^{\mathrm{f}}$ \\
\hline & $10 / 18$ & 0 & 319.363 & -11.279 & 10.126 & 32.424 & 1.735 \\
\hline & $100 / 177$ & 0 & 235.819 & 2.527 & 12.175 & 32.424 & 1.735 \\
\hline & $1,000 / 1,442$ & 0.1 & 77.306 & 5.218 & 5.948 & 16.835 & 0.202 \\
\hline & $5,000 / 5,773$ & 0.7 & 21.091 & $0.811(2)$ & $0.856(2)$ & 2.490 & 0.009 \\
\hline & $10,000 / 11,578$ & 1.5 & 17.333 & $0.811(2)$ & $0.839(2)$ & 1.892 & 0.028 \\
\hline & $50,000 / 92,682$ & 8.8 & 10.879 & $0.762(1)$ & $0.771(1)$ & 0.991 & 0.033 \\
\hline & $100,000 / 185,350$ & 13.9 & 9.243 & $0.632(1)$ & $0.639(1)$ & 0.727 & 0.023 \\
\hline & $500,000 / 742,754$ & 30.8 & 5.586 & $0.391(1)$ & $0.393(1)$ & 0.390 & 0.005 \\
\hline & $1,000,000 / 1,484,218$ & 37.1 & 4.795 & $0.330(0)$ & $0.332(0)$ & 0.362 & 0.004 \\
\hline & $5,000,000 / 5,907,228$ & 74.3 & 1.165 & $0.079(2)$ & $0.079(2)$ & 0.028 & -0.000 \\
\hline \multirow[t]{11}{*}{2.0} & $1 / 1$ & 0 & $640.056^{\mathrm{c}}$ & $-159.482^{\mathrm{d}}$ & 289.080 & $45.638^{\mathrm{e}}$ & $1.862^{\mathrm{f}}$ \\
\hline & $10 / 10$ & 0 & 337.263 & -3.392 & 19.484 & 45.638 & 1.862 \\
\hline & $100 / 122$ & 0.0 & 250.492 & 6.090 & 16.021 & 38.309 & 1.411 \\
\hline & $1,000 / 1,006$ & 0.1 & 105.265 & 5.589 & 7.036 & 21.727 & 0.132 \\
\hline & $5,000 / 8,118$ & 1.1 & 17.355 & $0.787(1)$ & $0.815(1)$ & 1.725 & -0.003 \\
\hline & $10,000 / 16,291$ & 2.1 & 14.555 & $0.860(1)$ & $0.878(1)$ & 1.338 & 0.012 \\
\hline & $50,000 / 65,172$ & 5.2 & 11.064 & $0.800(1)$ & $0.810(1)$ & 0.922 & 0.015 \\
\hline & $100,000 / 130,448$ & 8.4 & 9.410 & $0.655(1)$ & $0.662(1)$ & 0.695 & 0.009 \\
\hline & $500,000 / 521,578$ & 19.8 & 5.929 & $0.375(1)$ & $0.378(1)$ & 0.400 & 0.005 \\
\hline & $1,000,000 / 1,043,539$ & 28.0 & 4.820 & $0.306(0)$ & $0.308(0)$ & 0.314 & 0.002 \\
\hline & $5,000,000 / 8,190,854$ & 72.8 & 0.764 & $0.047(1)$ & $0.047(1)$ & 0.009 & -0.000 \\
\hline \multirow[t]{11}{*}{5.0} & $1 / 1$ & 0 & $730.244^{\mathrm{c}}$ & $-183.276^{\mathrm{d}}$ & 430.051 & $49.816^{\mathrm{e}}$ & $1.613^{\mathrm{f}}$ \\
\hline & $10 / 15$ & 0 & 310.757 & 4.700 & 21.059 & 49.816 & 1.613 \\
\hline & $100 / 151$ & 0.0 & 236.876 & 13.785 & 21.508 & 37.524 & 1.418 \\
\hline & $1,000 / 1,241$ & 0.2 & 70.879 & 6.966 & 7.491 & 5.154 & 0.144 \\
\hline & $5,000 / 9,977$ & 1.2 & 14.531 & $1.033(0)$ & $1.050(0)$ & 1.489 & 0.029 \\
\hline & $10,000 / 19,957$ & 2.2 & 12.550 & $1.039(0)$ & $1.050(0)$ & 1.156 & 0.029 \\
\hline & $50,000 / 79,866$ & 4.6 & 9.025 & $0.764(1)$ & $0.770(1)$ & 0.764 & 0.022 \\
\hline & $100,000 / 159,668$ & 7.6 & 7.495 & $0.580(1)$ & $0.584(1)$ & 0.584 & 0.013 \\
\hline & $500,000 / 639,593$ & 18.0 & 4.391 & $0.276(0)$ & $0.277(0)$ & 0.294 & 0.003 \\
\hline & $1,000,000 / 1,278,976$ & 22.0 & 3.682 & $0.238(0)$ & $0.239(0)$ & 0.259 & 0.003 \\
\hline & $5,000,000 / 5,099,863$ & 46.1 & 0.675 & $0.041(1)$ & $0.041(1)$ & 0.009 & -0.000 \\
\hline
\end{tabular}

a For each internuclear separation $R$, the $E_{\mathrm{var}}, E_{\mathrm{var}}+\Delta E^{(2)}$, and $E_{\mathrm{var}}+\Delta E_{\mathrm{r}}^{(2)}$ energies are reported as errors, in millihartree, relative to the extrapolated $E_{\mathrm{var}}+\Delta E_{\mathrm{r}}^{(2)}$ energy found using a linear fit based on the last four $E_{\mathrm{var}, k}+\Delta E_{\mathrm{r}, k}^{(2)}$ values leading to the largest CIPSI wave function obtained with $N_{\operatorname{det}(\mathrm{in})}=5,000,000$, plotted against the corresponding $\Delta E_{\mathrm{r}, k}^{(2)}$ corrections, following the procedure used in Ref. 72. These extrapolated $E_{\mathrm{var}}+\Delta E_{\mathrm{r}}^{(2)}$ energies at $R=R_{e}, 1.5 R_{e}, 2 R_{e}$, and $5 R_{e}$ are $-199.104422(6)$, -199.069043(1), -199.060152(8), and -199.059647(11) hartree, respectively, where the error bounds in parentheses correspond to the uncertainty associated with the linear fit. The error bounds for the $E_{\mathrm{var}}+\Delta E^{(2)}$ and $E_{\mathrm{var}}+\Delta E_{\mathrm{r}}^{(2)}$ energies obtained at the various values of $N_{\operatorname{det}(i n)}$ reflect on the semi-stochastic design of the $\mathcal{V}_{\text {ext }}^{(k)}$ spaces discussed in the main text, but they ignore the uncertainties characterizing the reference $E_{\mathrm{var}}+\Delta E_{\mathrm{r}}^{(2)}$ energies obtained in the above extrapolation procedure.

b The $\mathrm{CC}(P)$ and $\mathrm{CC}(P ; Q)$ energies are reported as errors relative to CCSDT, in millihartree. The total CCSDT energies at $R=R_{e}$, $1.5 R_{e}, 2 R_{e}$, and $5 R_{e}$ are $-199.102796,-199.065882,-199.058201$, and -199.058586 hartree, respectively.

c Equivalent to RHF.

d Equivalent to the second-order MBPT energy using the Epstein-Nesbet denominator.

e Equivalent to CCSD.

f Equivalent to CR-CC(2,3). 
TABLE III. Convergence of the $\mathrm{CC}(P)$ and $\mathrm{CC}(P ; Q)$ energies toward CCSDT, alongside the variational and perturbatively corrected CIPSI energies, for the $\mathrm{F}_{2} / \mathrm{cc}$-pVTZ molecule in which the $\mathrm{F}-\mathrm{F}$ bond length $R$ was fixed at $2 R_{e}$, where $R_{e}=2.66816$ bohr is the equilibrium geometry. For each value of the wave function termination parameter $N_{\operatorname{det}(\text { in) }}$, the $P$ space used in the $\mathrm{CC}(P)$ calculations consisted of all singles and doubles and a subset of triples contained in the final $\left|\Psi^{(\mathrm{CIPSI})}\right\rangle$ state of the underlying CIPSI run, whereas the $Q$ space needed to compute the corresponding $\mathrm{CC}(P ; Q)$ correction $\delta(P ; Q)$ was defined as the remaining triples absent in $\left|\Psi^{(\mathrm{CIPSI})}\right\rangle$. In all post-RHF calculations, the two lowest-lying core orbitals were frozen and the spherical components of $d$ and $f$ orbitals were employed throughout. Each CIPSI run was initiated from the RHF reference determinant and the MBPT-based stopping parameter $\eta$ was set at $10^{-6}$ hartree. The input parameter $f$ controlling the CIPSI wave function growth was set at the default value of 2 .

\begin{tabular}{|c|c|c|c|c|c|c|}
\hline$N_{\operatorname{det}(\text { in })} / N_{\operatorname{det}(\text { out })}$ & $\%$ of triples & $E_{\mathrm{var}}{ }^{\mathrm{a}}$ & $E_{\mathrm{var}}+\Delta E^{(2) \mathrm{a}}$ & $E_{\mathrm{var}}+\Delta E_{\mathrm{r}}^{(2) \mathrm{a}}$ & $\mathrm{CC}(P)^{\mathrm{b}}$ & $\mathrm{CC}(P ; Q)^{\mathrm{b}}$ \\
\hline $1 / 1$ & 0 & $758.849^{\mathrm{C}}$ & $-165.740^{\mathrm{d}}$ & 340.460 & $62.819^{\mathrm{e}}$ & $4.254^{\mathrm{f}}$ \\
\hline $10 / 18$ & 0 & 441.567 & -0.554 & 31.337 & 62.819 & 4.254 \\
\hline $100 / 156$ & 0.00 & 393.749 & 6.420 & 28.790 & 58.891 & 3.683 \\
\hline $1,000 / 1,277$ & 0.01 & 253.172 & $13.595(0)$ & 20.323 & 42.564 & 1.579 \\
\hline $5,000 / 5,118$ & 0.03 & 123.591 & $10.874(1)$ & $12.149(1)$ & 18.036 & 0.345 \\
\hline 10,000 / 10,239 & 0.06 & 73.122 & $7.202(5)$ & $7.636(5)$ & 11.439 & 0.198 \\
\hline $50,000 / 82,001$ & 0.84 & 29.674 & $3.371(2)$ & $3.428(2)$ & 4.898 & 0.061 \\
\hline $100,000 / 163,866$ & 1.58 & 27.002 & $3.068(2)$ & $3.113(2)$ & 4.157 & 0.049 \\
\hline $500,000 / 655,859$ & 3.75 & 22.301 & $2.517(1)$ & $2.547(1)$ & 3.111 & 0.014 \\
\hline $1,000,000 / 1,311,633$ & 5.58 & 20.244 & $2.292(1)$ & $2.316(1)$ & 2.739 & 0.009 \\
\hline $5,000,000 / 5,253,775$ & 13.3 & 14.499 & $1.645(1)$ & $1.657(1)$ & 1.866 & -0.015 \\
\hline
\end{tabular}

a The $E_{\mathrm{var}}, E_{\mathrm{var}}+\Delta E^{(2)}$, and $E_{\mathrm{var}}+\Delta E_{\mathrm{r}}^{(2)}$ energies are reported as errors, in millihartree, relative to the extrapolated $E_{\mathrm{v} a r}+\Delta E_{\mathrm{r}}^{(2)}$ energy found using a linear fit based on the last four $E_{\mathrm{var}, k}+\Delta E_{\mathrm{r}, k}^{(2)}$ values leading to the largest CIPSI wave function obtained with $N_{\operatorname{det}(\text { in })}=5,000,000$, plotted against the corresponding $\Delta E_{\mathrm{r}, k}^{(2)}$ corrections, following the procedure used in Ref. 72 . The extrapolated $E_{\mathrm{var}}+\Delta E_{\mathrm{r}}^{(2)}$ energy is $-199.242119(59)$ hartree, where the error bounds in parentheses correspond to the uncertainty associated with the linear fit. The error bounds for the $E_{\mathrm{var}}+\Delta E^{(2)}$ and $E_{\mathrm{var}}+\Delta E_{\mathrm{r}}^{(2)}$ energies obtained at the various values of $N_{\mathrm{det}(\mathrm{in})}$ reflect on the semi-stochastic design of the $\mathcal{V}_{\text {ext }}^{(k)}$ spaces discussed in the main text, but they ignore the uncertainties characterizing the reference $E_{\mathrm{var}}+\Delta E_{\mathrm{r}}^{(2)}$ energy obtained in the above extrapolation procedure.

b The $\mathrm{CC}(P)$ and $\mathrm{CC}(P ; Q)$ energies are reported as errors relative to CCSDT, in millihartree. The total CCSDT energy is -199.238344 hartree.

c Equivalent to RHF.

d Equivalent to the second-order MBPT energy using the Epstein-Nesbet denominator.

e Equivalent to CCSD.

f Equivalent to CR-CC(2,3). 
TABLE IV. Convergence of the $\mathrm{CC}(P)$ and $\mathrm{CC}(P ; Q)$ energies toward CCSDT, alongside the variational and perturbatively corrected CIPSI energies, for the reactant (R) and transition-state (TS) species involved in the automerization of cyclobutadiene, as described by the cc-pVDZ basis set, and for the corresponding barrier height. The R and TS geometries, optimized using the MR-AQCC approach, were taken from Ref. 95. For each value of the wave function termination parameter $N_{\text {det(in) }}$, the $P$ space used in the $\mathrm{CC}(P)$ calculations consisted of all singles and doubles and a subset of triples contained in the final $\left|\Psi^{(\mathrm{CIPSI})}\right\rangle$ state of the underlying CIPSI run, whereas the $Q$ space needed to compute the corresponding $\mathrm{CC}(P ; Q) \operatorname{correction} \delta(P ; Q)$ was defined as the remaining triples absent in $\left|\Psi^{(\mathrm{CIPSI})}\right\rangle$. In all post-RHF calculations, the four lowest-lying core orbitals were frozen and the spherical components of $d$ orbitals were employed throughout. Each CIPSI run was initiated from the RHF reference determinant and the MBPT-based stopping parameter $\eta$ was set at $10^{-6}$ hartree. The input parameter $f$ controlling the CIPSI wave function growth was set at the default value of 2 .

\begin{tabular}{|c|c|c|c|c|c|c|c|}
\hline Species & $N_{\operatorname{det}(\text { in })} / N_{\operatorname{det}(\text { out })}$ & $\%$ of triples & $E_{\text {var }}{ }^{\mathrm{a}}$ & $E_{\mathrm{var}}+\Delta E^{(2) \mathrm{a}}$ & $E_{\mathrm{var}}+\Delta E_{\mathrm{r}}^{(2) \mathrm{a}}$ & $\mathrm{CC}(P)^{\mathrm{b}}$ & $\mathrm{CC}(P ; Q)^{\mathrm{b}}$ \\
\hline \multirow[t]{8}{*}{$\overline{\mathrm{R}}$} & $1 / 1$ & 0 & $598.120^{\mathrm{C}}$ & $-83.736^{\mathrm{d}}$ & 120.809 & $26.827^{\mathrm{e}}$ & $0.848^{\mathrm{f}}$ \\
\hline & $50,000 / 55,653$ & 0.0 & 121.880 & $26.065(182)$ & $28.096(178)$ & 25.468 & 0.678 \\
\hline & $100,000 / 111,321$ & 0.1 & 109.688 & $23.819(163)$ & $25.397(160)$ & 22.132 & 0.382 \\
\hline & $500,000 / 890,582$ & 1.2 & 93.413 & $19.049(141)$ & $20.167(139)$ & 16.260 & 0.267 \\
\hline & $1,000,000 / 1,781,910$ & 2.0 & 89.989 & $18.322(137)$ & $19.348(135)$ & 15.359 & 0.251 \\
\hline & $5,000,000 / 7,125,208$ & 7.9 & 78.122 & 16.311(123) & $17.045(122)$ & 10.794 & 0.150 \\
\hline & $10,000,000 / 14,253,131$ & 11.8 & 73.250 & $15.514(115)$ & $16.146(114)$ & 9.632 & 0.127 \\
\hline & $15,000,000 / 28,493,873$ & 25.8 & 60.872 & $12.842(96)$ & $13.260(95)$ & 4.817 & 0.046 \\
\hline \multirow[t]{8}{*}{$\mathrm{TS}$} & $1 / 1$ & 0 & $632.707^{\mathrm{c}}$ & $-102.816^{\mathrm{d}}$ & 282.246 & $47.979^{\mathrm{e}}$ & $14.636^{\mathrm{f}}$ \\
\hline & $50,000 / 56,225$ & 0.0 & 146.895 & $45.357(180)$ & 47.696(176) & 42.132 & 9.563 \\
\hline & $100,000 / 112,481$ & 0.1 & 130.832 & $36.716(183)$ & $38.673(179)$ & 31.723 & 3.507 \\
\hline & $500,000 / 899,770$ & 1.0 & 93.288 & $18.106(139)$ & $19.251(137)$ & 14.742 & 0.432 \\
\hline & $1,000,000 / 1,800,183$ & 1.6 & 89.049 & $17.458(142)$ & $18.482(140)$ & 13.645 & 0.412 \\
\hline & $5,000,000 / 7,195,780$ & 5.4 & 78.472 & $15.587(124)$ & $16.346(123)$ & 10.720 & 0.260 \\
\hline & $10,000,000 / 14,400,744$ & 9.7 & 71.784 & 14.397(114) & $15.016(113)$ & 8.358 & 0.155 \\
\hline & $15,000,000 / 28,793,512$ & 15.2 & 63.375 & $12.587(102)$ & $13.058(101)$ & 7.080 & 0.108 \\
\hline \multirow[t]{8}{*}{ Barrier } & $1 / 1 ; 1$ & $0 ; 0$ & $21.703^{\mathrm{c}}$ & $-11.973^{\mathrm{d}}$ & 101.303 & $13.274^{\mathrm{e}}$ & $8.653^{\mathrm{f}}$ \\
\hline & $50,000 / 55,653 ; 56,225$ & $0.0 ; 0.0$ & 15.697 & $12.106(161)$ & $12.299(157)$ & 10.457 & 5.576 \\
\hline & $100,000 / 111,321 ; 112,481$ & $0.1 ; 0.1$ & 13.268 & $8.093(154)$ & $8.331(151)$ & 6.018 & 1.961 \\
\hline & $500,000 / 890,582 ; 899,770$ & $1.2 ; 1.0$ & -0.079 & $-0.592(124)$ & $-0.574(122)$ & -0.953 & 0.104 \\
\hline & $1,000,000 / 1,781,910 ; 1,800,183$ & $2.0 ; 1.6$ & -0.590 & $-0.542(124)$ & $-0.544(122)$ & -1.075 & 0.101 \\
\hline & $5,000,000 / 7,125,208 ; 7,195,780$ & $7.9 ; 5.4$ & 0.220 & $-0.454(110)$ & $-0.439(109)$ & -0.047 & 0.069 \\
\hline & $10,000,000 / 14,253,131 ; 14,400,744$ & $11.8 ; 9.7$ & -0.920 & $-0.701(102)$ & $-0.710(100)$ & -0.800 & 0.017 \\
\hline & $15,000,000 / 28,493,873 ; 28,793,512$ & $25.8 ; 15.2$ & 1.571 & $-0.159(88)$ & $-0.127(87)$ & 1.420 & 0.039 \\
\hline
\end{tabular}

a For each of the two species, the $E_{\mathrm{var}}, E_{\mathrm{var}}+\Delta E^{(2)}$, and $E_{\mathrm{var}}+\Delta E_{\mathrm{r}}^{(2)}$ energies are reported as errors, in millihartree, relative to the extrapolated $E_{\mathrm{var}}+\Delta E_{\mathrm{r}}^{(2)}$ energy found using a linear fit based on the last four $E_{\mathrm{var}, k}+\Delta E_{\mathrm{r}, k}^{(2)}$ values leading to the largest CIPSI wave function obtained with $N_{\operatorname{det}(\text { in })}=15,000,000$, plotted against the corresponding $\Delta E_{\mathrm{r}, k}^{(2)}$ corrections, following the procedure used in Ref. 72. These extrapolated $E_{\mathrm{var}}+\Delta E_{\mathrm{r}}^{(2)}$ energies for the R and TS species are -154.249292(314) and -154.235342(321) hartree, respectively, where the error bounds in parentheses correspond to the uncertainty associated with the linear fit. The error bounds for the $E_{\mathrm{var}}+\Delta E^{(2)}$ and $E_{\mathrm{var}}+\Delta E_{\mathrm{r}}^{(2)}$ energies obtained at the various values of $N_{\operatorname{det}(\mathrm{in})}$ reflect on the semi-stochastic design of the $\mathcal{V}_{\text {ext }}^{(k)}$ spaces discussed in the main text, but they ignore the uncertainties characterizing the reference $E_{\mathrm{var}}+\Delta E_{\mathrm{r}}^{(2)}$ energies obtained in the above extrapolation procedure. The $E_{\mathrm{var}}, E_{\mathrm{var}}+\Delta E^{(2)}$, and $E_{\mathrm{var}}+\Delta E_{\mathrm{r}}^{(2)}$ barrier heights are reported as errors, in kcal/mol, relative to the reference value of $8.753(0) \mathrm{kcal} / \mathrm{mol}$ obtained using the extrapolated $E_{\mathrm{var}}+\Delta E_{\mathrm{r}}^{(2)}$ energies of the R and TS species.

b The $\mathrm{CC}(P)$ and $\mathrm{CC}(P ; Q)$ energies of the $\mathrm{R}$ and TS species are reported as errors relative to CCSDT, in millihartree. The total CCSDT energies of the R and TS species are -154.244157 and -154.232002 hartree, respectively. The $\mathrm{CC}(P)$ and $\mathrm{CC}(P ; Q)$ barrier heights are reported in $\mathrm{kcal} / \mathrm{mol}$ relative to the CCSDT value of $7.627 \mathrm{kcal} / \mathrm{mol}$.

c Equivalent to RHF.

d Equivalent to the second-order MBPT energy using the Epstein-Nesbet denominator.

e Equivalent to CCSD.

f Equivalent to CR-CC $(2,3)$. 

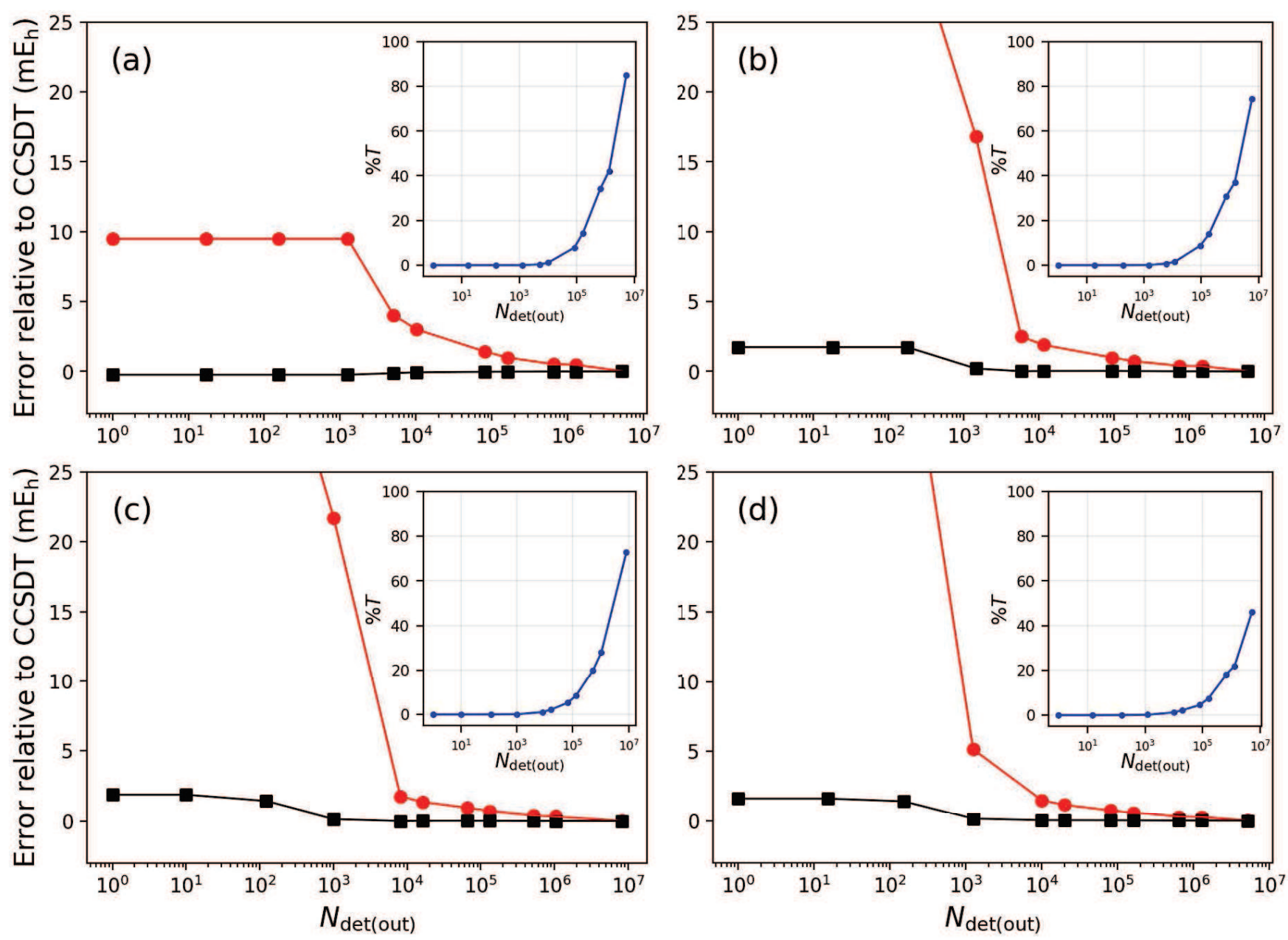

FIG. 1. Convergence of the $\mathrm{CC}(P)$ (red lines and circles) and $\mathrm{CC}(P ; Q)$ (black lines and squares) energies toward their CCSDT parents as functions of the actual numbers of determinants, $N_{\operatorname{det}(\text { out })}$, defining the sizes of the final wave functions $\left|\Psi^{(\mathrm{CIPSI})}\right\rangle$ generated in the underlying CIPSI runs, for the $\mathrm{F}_{2} / \mathrm{cc}-\mathrm{pVDZ}$ molecule in which the $\mathrm{F}-\mathrm{F}$ bond length $R$ was set at (a) $R_{\mathrm{e}}$, (b) $1.5 R_{\mathrm{e}}$, (c) $2 R_{\mathrm{e}}$, and (d) $5 R_{\mathrm{e}}$, where $R_{e}=2.66816 \mathrm{bohr}$ is the equilibrium geometry. The $P$ spaces used in the CC( $\left.P\right)$ calculations consisted of all singles and doubles and subsets of triples contained in the final $\left|\Psi^{(\mathrm{CIPSI})}\right\rangle$ states of the underlying CIPSI runs, whereas the $Q$ spaces needed to compute the corresponding $\operatorname{CC}(P ; Q)$ corrections $\delta(P ; Q)$ were defined as the remaining triples absent in $\left|\Psi^{(\mathrm{CIPSI})}\right\rangle$. The insets show the percentages of triples captured by the CIPSI runs as functions of $N_{\text {det(out) }}$. 

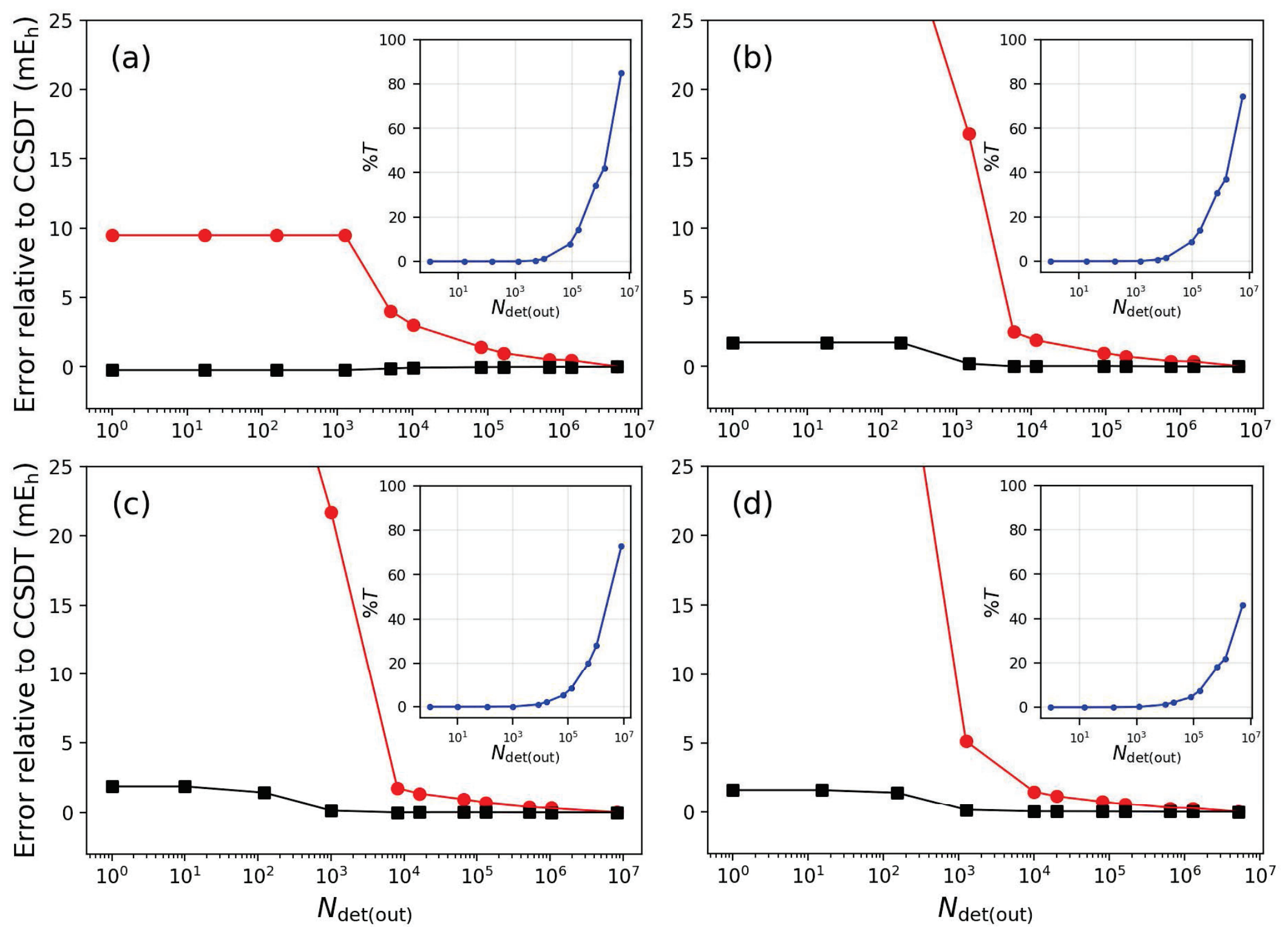